\title{
Two-Dimensional and Three-Dimensional Simulations of Oscillatory Flow around a Circular Cylinder
}

\author{
Hongwei $\mathrm{An}^{1 \mathrm{a}}$, Liang Cheng ${ }^{\mathrm{a}}$ and Ming Zhao ${ }^{\mathrm{b}}$ \\ School of Civil and Resource Engineering, the University of Western Australia \\ 35 Stirling Highway, WA 6009, Australia \\ School of Computing, Engineering and Mathematics, University of Western Sydney \\ Locked Bag 1797, Penrith NSW 2751, Australia
}

\begin{abstract}
Sinusoidal oscillatory flow around a circular cylinder is simulated using a twodimensional (2D) finite element model and a three-dimensional (3D) finite element model respectively at a constant Reynolds number $(\mathrm{Re})$ of 2000 and seven Keulegan-Carpenter $(K C)$ numbers of 1, 2, 5, 10, 17.5, 20 and 26.2. The identical finite element method is used in both the $2 \mathrm{D}$ and $3 \mathrm{D}$ models. The purpose of this study is to investigate the feasibility of a 2D model for simulating a seemingly 3D flow in terms of fundamental flow characteristics and hydrodynamic forces on the cylinder. It is found that the vortex structures predicted by the 2D model agree qualitatively with those by 3D model for the flow cases where strong correlations exist along the span-wise direction (e.g. $K C=10,17.5$ and 26.2). Three vortex shedding modes are reproduced by both $2 \mathrm{D}$ and $3 \mathrm{D}$ models at $K C=20$, which is close to the critical $K C$ number between double-pair and three-pair regimes. The time histories of hydrodynamic force predicted by the two models agree with each other at $K C=20$ from the mode-average point of view. The predicted Morison force coefficients by the 2D model are found to be within $18 \%$ different from of those predicted using the 3D model for most of the cases investigated in this study. It is demonstrated that a 2D model captures the majority of the genuine flow structures and hydrodynamic load features of a circular cylinder in an oscillatory flow.
\end{abstract}

\section{Introduction}

Oscillatory flow around a circular cylinder has been of significant interests in both research and engineering communities for many years due to its rich flow features and engineering relevance. The flow has been widely used to approximate wave-induced water motions around cylindrical structures in offshore engineering where structures are designed

\footnotetext{
${ }^{1}$ Corresponding author. Tel. +61864887225

Email address: hongwei.an@uwa.edu.au
} 
against wave forces. An undisturbed oscillatory flow (far away from the cylinder) is often defined as $u(t)=U_{m} \sin (2 \pi t / T)$, where $U_{m}$ and $T$ are the amplitude and the period of oscillatory velocity, respectively. It is well known that oscillatory flow characteristics around a circular cylinder is governed by $K C$ number $\left(K C=U_{m} T / D\right)$ and Reynolds number $\left(\operatorname{Re}=U_{m} D / v\right)$ or frequency number $\left(\beta=\operatorname{Re} / K C=D^{2} / v T\right)$, where $D$ is the diameter of the cylinder, $v$ is the kinematic viscosity of fluid.

This topic can be examined through different setup of coordinate systems. In most of the experimental research, the cylinder was given oscillatory motion in still water (Williamson 1985, Tatsuno and Bearman 1990, Lam and Dai 2010), where the coordinate system was fixed with far field water. In numerical simulation works, it is more convenient to simulate an oscillating flow past a stationary cylinder (Justesen 1991, Elston et al. 2006 and An et al. 2011), where the coordinate system was fixed with the cylinder in the numerical simulations to avoid dealing with mesh deformation. The results from the two different research methods should be comparable. The difference between an oscillating cylinder and a fixed cylinder is due to the fact that in the case of a fixed cylinder experiences the Froude-Krylov force due to the pressure gradient along the oscillation direction. The inertial coefficient $C_{M}$ that appears in the Morison equation can be written as $C_{M}=1+C_{a}$, where $C_{a}$ is the added mass coefficient and 1 represents the contribution of the Froude-Krylov force. In the case of an oscillating cylinder in still water the Froude-Krylov force vanishes. In this work, it is referred to as oscillatory flow around a circular cylinder in the discussions.

Oscillatory flow around a circular cylinder involves many complicated flow phenomena, such as boundary layer separation, vortex shedding, and flow reversal, etc. The flow shows 3D effect under most of flow conditions in practical engineering. A comprehensive review about the interaction between oscillatory flow and cylindrical structure is given by Sumer and Fredsøe (2002). Large amount of experimental investigations about oscillatory flow around a cylinder have been carried out to investigate the flow features. It was found that the transition from $2 \mathrm{D}$ to $3 \mathrm{D}$ is dependent on both $K C$ and $\beta$. Oscillatory flow around a circular cylinder is 2D only for very small values of $K C$. The onset of 3D flow structures was investigated by Honji (1985), Tatsuno and Bearman (1990) and Sarpkaya (1986). Sarpkaya (2002) proposed an empirical equation for the critical $K C$ number based on experimental results:

$$
K_{c r} \beta^{2 / 5}=12.5
$$

where $K_{c r}$ represents the critical $K C$ number above which the oscillatory flow becomes 3D. 
Williamson (1986) visualized the flow structures around an oscillatory cylinder in still water by means of particles on free surface at $\beta=255$. Six vortex shedding regimes were found in the range of $1<K C<40$. Williamson (1985) found that the lift oscillations become less repeatable at large values of $K C$, suggesting the possibility of the co-existence of multiple vortex shedding regimes. Obasaju (1988) measured the inline and lift forces of a circular cylinder in oscillatory flow and found that the force coefficients were dependent on both $K C$ and $\beta$. Lam et al. (2010) re-visited the oscillatory flow in the range of $K C=8 \sim 36$ and $\mathrm{Re}=2400$. Detailed description about migration, stretching, and splitting of vortices around the cylinder was given.

Extensive numerical simulations have been undertaken to investigate oscillatory flow around a circular cylinder during the last three decades. Most of the numerical studies conducted so far are two-dimensional (Justesen 1991, Lin et al 1996, Saghafian et al. 2003). Detailed discussion about the force coefficients, flow structures, pressure distribution and time histories of forces were given by Justesen (1991) in the range of $0.1<K C<26$ and $\beta=196$, 483 and 1035. Justesen (1991) also compared the numerical results of drag and inertia coefficients $\left(C_{D}\right.$ and $\left.C_{M}\right)$ with the experimental data of Obasaju (1988). It was found that the numerical model tended to under-predict $C_{D}$ and $C_{M}$ slightly in the range of $1<K C<12(\beta=$ 196). A good comparison was found for $C_{D}$ in the range of $12<\mathrm{KC}<26$, but $C_{M}$ was also under-predicted in this range. Saghafian et al. (2003) simulated oscillatory flow at high $\beta$ numbers $(\beta=1035$ and 11240) by solving 2D Reynolds-Averaged Navier-Stokes (RANS) equations with a high order $k-\varepsilon$ turbulent model. It was shown that the calculated drag and inertia coefficients agree with the experimental data reasonably well.

With the rapid increase of computational power in recent years, a number of 3D numerical simulations have been carried out to investigate oscillatory flow around a circular cylinder. Much improved understanding of flow mechanisms has been achieved through the 3D simulations. For examples, Honji instability (Honji 1981) was numerically captured by Zhang and Dalton (1999) and An et al. (2011). Elston et al. (2006) simulated oscillatory flow in the range of $K C=0 \sim 10, \beta=0 \sim 100$ and investigated the primary and secondary instabilities of oscillatory flow around a circular cylinder. It was found that the primary instability of the oscillatory flow is $3 \mathrm{D}$ (Honji instability) for $50<\beta<100$, which could not be captured by 2D models. Zhao and Cheng (2011) carried out a number of 3D simulations of oscillatory flow around an inclined cylinder in the range of $K C=6.75 \sim 30$ and a constant $\mathrm{Re}$ 
number of 2000. It was found the hydrodynamic forces follow the cosine law for flow incidence angle up to $45^{\circ}$.

Although 3D simulations of various fluid flow problems have increased significantly in recent years due to the rapid increase in computing power, 2D simulations are still widely used in engineering applications and will remain so for a period of time. This is mainly because of the low computational costs and quick turnover times of $2 \mathrm{D}$ runs. This is especially important in front engineering phase when project schedules are tight. In order to use 2D models with confidence, it is necessary to evaluate computational errors induced by $2 \mathrm{D}$ simulations. So far such an evaluation is unavailable for oscillatory flow around a circular cylinder, to the best knowledge of the authors. The present study is motivated primarily by this issue.

In this study, sinusoidal oscillatory flow past a circular cylinder is investigated using both 2D and 3D models at Re number of 2000 and $K C$ number of 1, 2, 5, 10, 17.5, 20 and 26.2. The Petrov-Galerkin finite element method is used to solve the NS equations in this study.

\section{Numerical method}

In both 2D and 3D numerical models, Navier-Stokes equations are solved by the PetrovGalerkin finite element method (PG-FEM) developed by Brooks and Hughes (1982). A definition sketch of 2D oscillatory flow around a circular cylinder is shown in Figure 2(a). A Cartesian coordinate system is fixed at the centre of the circular cylinder and $y$-axis is perpendicular to the oscillatory flow direction. The oscillatory flow is governed by NavierStokes equations given as:

$$
\begin{aligned}
& u_{i, t}+u_{j} u_{i, j}+p_{, j}-\frac{1}{\operatorname{Re}} u_{i, j j}=0 \\
& u_{i, i}=0
\end{aligned}
$$

where $u_{i}$ represents velocity component in the $x_{i}$-direction, $\left(x_{1}, x_{2}, x_{3}\right)=(x, y, z)$, $\left(u_{1}, u_{2}, u_{3}\right)=(u, v, w)$, the subscripts in $f_{, t}$ and $f_{, i}$ represent the derivatives of $f$ with respect to time $t$ and $x_{i}$ respectively, and $p$ is the pressure. The initial condition is

$$
(u, v, w)=0
$$

Non-slip boundary condition is given on surface of the cylinder. On the inlet boundary, velocity is specified as:

$$
(u, v, w)=(U(t), 0,0)
$$


where the free stream velocity is given as:

$$
U(t)=U_{m} \sin (2 \pi t / T)
$$

Free slip boundary condition is implemented on top and bottom boundaries. At the outflow boundary, the pressure is set to be zero. At the two ends of the cylinder, periodic boundary condition is applied.

Petrov-Galerkin finite element method is employed for discretizing the Navier-Stokes equations. In the Petrov-Galerkin formulation the standard Galerkin weighting functions are modified by adding a streamline upwind perturbation, which acts only in the flow direction (Brooks and Hughes 1982). This FEM model has been demonstrated to be well suited to simulate three-dimensional flow around a circular cylinder (Zhao et al. 2011, An et al. 2011 and Yang et al. 2014). A constant Re value of 2000 is used in all the numerical simulation. The NS equations are solved directly (DNS) to simulate the oscillatory flow around the cylinder considering the relatively low Re number condition and no turbulence model is applied in the simulations. A Fortran language code developed by Zhao et al. (2011) is used to simulate the flows.

A rectangular computational domain of $60 D$ in length and $40 D$ in width is used for simulating the oscillatory flow. Figure 3 shows a typical 2D finite element mesh near the cylinder. Structured four-node quadrilateral elements are used to discretize the domain. The cylinder perimeter is discretized by 80 nodes. In order to determine the appropriate mesh density, the mesh dependency in the $x-y$ plane is examined first. Three two-dimensional meshes in the $x-y$ plane are examined and the mesh properties are listed in table 1 . The minimum element size in the radial direction next to the cylinder surface $\left(\Delta_{1}\right)$ is $0.001 \mathrm{D}$. Such a value of $\Delta_{1}$ results in the non-dimensional mesh size $y^{+}$smaller than 1 for all the flow simulated in the present study. The $y^{+}$is defined as $y^{+}=u_{f} \Delta_{1} / v$, where $u_{f}$ is the friction velocity. The same $\Delta_{1}$ value was used for the three meshes. The cylinder surface was discretised with 60, 80 and 100 uniform elements for the three meshes, respectively. The nondimensional computational time step size is $\Delta t=0.001$.

In the mesh dependency test, a flow with $K C=10$ was used. The phase averaged (over $10 T)$ pressure coefficient $\left(C_{p}\right)$ on the cylinder surface at peak velocity $(\phi=\pi / 2, \phi$ is the phase angle of the oscillatory flow) is examined as shown in Figure 4 (a). The definition of angle $\alpha$ is given in Figure 2 (a). It can be seen maximum difference of pressure coefficient between 
mesh 1 and mesh 2 is about $7 \%$. The difference of $C_{p}$ between mesh 2 and mesh 3 is less than $2 \%$, although the total element number in mesh 3 is $25 \%$ more than that in mesh2. Therefore the mesh2 was selected to carry out three-dimensional mesh dependency.

Figure 2 (b) shows a definition sketch of the 3D coordinate system and computational domain. The 2D mesh is replicated along the spanwise direction to generate the $3 \mathrm{D}$ mesh. In the three-dimensional mesh dependency study, only the spanwise density was examined and the mesh density in the $\mathrm{x}-\mathrm{y}$ plane remained the same as mesh2 in the $2 \mathrm{D}$ dependency test. Three spanwise mesh sizes $(0.05 \mathrm{D}, 0.1 \mathrm{D}$ and $0.15 \mathrm{D})$ are examined and the span length of the cylinder is set at $19.6 \mathrm{D}$ at $\mathrm{KC}=10$. The pressure coefficient distribution along the cylinder surface is plotted in Figure 4 (b). The two-dimensional result with mesh2 is also plotted for a reference. The simulated results show a good convergence trend. There is a $50 \%$ increase of finite element number from mesh 5 to mesh 6 , but the simulation results show very minor difference in term of pressure distribution and main flow structure around the cylinder. Due to the main purpose of this work was to compare the hydrodynamic force from $2 \mathrm{D}$ and $3 \mathrm{D}$ simulations, mesh 5 was used for three-dimensional simulation of other KC numbers in this study to save computational time.

It can be seen from Figure 4 (b) that all the four meshes give pretty close pressure coefficient on the upstream surface $\left(0^{\circ}<\alpha<90^{\circ}\right.$ and $\left.270^{\circ}<\alpha<360^{\circ}\right)$. For the downstream surface $\left(90^{\circ}<\alpha<270^{\circ}\right)$, the difference between the $2 \mathrm{D}$ and $3 \mathrm{D}$ results is much more obvious. This is because the $3 \mathrm{D}$ behaviour of the flow structure on the upstream side surface is relatively weak in this case. Therefore, even the two-dimensional model can capture the upstream pressure distribution with high accuracy. However the downstream flow has much stronger three-dimensional features than that at upstream. As an example, the mesh5 results (spanwise vorticity iso-surfaces, $\omega_{z}= \pm 5$ ) at $\mathrm{t} / \mathrm{T}=10.25$ are shown in Figure 5.

Two different spanwise dimensions were used in this study for different $\mathrm{KC}$ numbers. At relative low $K C$ numbers $(K C=1,2$ and 5), the length of the cylinder is chosen as $8 \times \mathrm{D}$ based on the observations that the wave length of periodic 3D flow structures along the spanwise direction are approximately $0.5 \times \mathrm{D}$ to $1 \times \mathrm{D}$ at relatively low $K C$ number (Honji 1981 and Sarpkaya 1986). A shorter spanwise size has been used in numerical simulation for similar flow conditions (An et al. 2011 and Yang et al. 2014). 8×D is sufficient for $\mathrm{KC}=1$ and 2. KC $=5$ is the transition between the symmetric attached vortex pair regime and the one-pair shedding regime (Williamson 1985 and Obasaju et al. 1988). Obasaju has demonstrated that a 
very weak spanwise correlation is normally observed for a transition flow between two different oscillatory flow shedding regimes. It indicates that transition flow has a strong 3D feature, but without regular spanwise coherent flow structures. Therefore a span length of $8 \times \mathrm{D}$ is also used for $\mathrm{KC}=5$. At relatively large $K C$ number $(K C=10,17.5,20$ and 26.5), a larger cylinder length of $19.6 \times \mathrm{D}$ is chosen to resolve large correlation lengths (about $4 \times \mathrm{D}$ to $5 \times \mathrm{D}$, Obasaju et al. 1988). This span length has been used by Zhao et al. (2011) to investigate inclined oscillatory flow around a circular cylinder and has been demonstrated to be sufficient to capture the spanwise direction flow structures.

\section{Numerical results}

In this work, a 2D and a 3D finite element models are used to simulate oscillatory flow past a circular cylinder at a constant Re number of 2000 and seven $\mathrm{KC}$ numbers of $K C=1,2,5,10$, 17.5, 20 and 26.2. Detailed comparison about flow structure and hydrodynamic forces obtained from 2D and 3D simulations are presented. The 2D and 3D numerical results are compared through vortex shedding patterns, vorticity strength and hydrodynamic forces. Section 3.1 3.7 provides detailed descriptions about flow structures and force time histories in each $\mathrm{KC}$ number and Section 3.8 summarizes the Morison force coefficients.

\section{1 $K C=1$}

For $K C=1$ and $\mathrm{Re}=2000,3 \mathrm{D}$ instability of flow around the cylinder is found. Figure 6 shows the vorticity iso-surfaces of $\omega_{x}= \pm 0.5, \omega_{y}= \pm 0.5$ and $\omega_{z}= \pm 0.5$ at the instant of $t / T=$ $30.25(\phi=0.5 \pi)$, which corresponds to the maximum free stream velocity. The vorticity components are defined as $\omega_{x}=\partial w / \partial y-\partial v / \partial z, \omega_{y}=\partial u / \partial z-\partial w / \partial x$ and $\omega_{z}=\partial v / \partial x-\partial u / \partial y$. It is observed that regular 3D rid-shaped vortex tubes are distributed along the span-wise direction of the cylinder, which is a typical feature of Honji vortices (Honji 1981). More detailed description about the flow regime can be found in An et al. (2013). The primary aim here is to compare the 2D and 3D simulations.

The amplitudes of $\omega_{x} \omega_{y}$ and $\omega_{z}$ are 2.5, 1.5 and 202, respectively. The peak value of the vorticity happens in the flow boundary layer on the cylinder surface (within the first layer of mesh elements next to the cylinder surface). The amplitudes of $\omega_{x}$ and $\omega_{y}$ are much lower than that of $\omega_{z}$, indicating the three-dimensionality of this case is weak. A comparison of the flow structure in the $\mathrm{x}-\mathrm{y}$ plane is given in Figure 7. It is found that the $3 \mathrm{D}$ result shows slight asymmetry with respect to the $x$-axis, while the $2 \mathrm{D}$ result is symmetric. The peak value of $\omega_{z}$ in the $2 \mathrm{D}$ simulation is 190 , which is very close to the value observed in the $3 \mathrm{D}$ simulation. 
The inline and lift forces at a section of the cylinder are calculated by integrating the pressure and shear stress over the circumference of the cylinder. The non-dimensional sectional force is defined as $C_{x}(z)=F_{x}(z) /\left(\rho D U_{m}^{2} / 2\right), C_{y}(z)=F_{y}(z) /\left(\rho D U_{m}^{2} / 2\right)$, where $F_{x}(z)$ and $F_{y}(z)$ are hydrodynamic force components in the $x$ - and $y$-axis directions, respectively. As the amplitude of $\omega_{x}$ and $\omega_{y}$ are much lower than that of $\omega_{z}$, the variation of inline forces along span-wise direction is negligibly small. Figure 8 shows a comparison of inline forces calculated by 2D and 3D models. Good agreement between the results calculated by the twomodels is found. It is also obvious that $C_{x}$ is in phase with flow acceleration, indicating an inertia dominated condition in this case. The asymmetric vortex structures shown in Figure 7 (a) lead to non-zero lift force $C_{y}$, but with an amplitude three orders lower than that of inline forces $C_{x}$. Zero lift force is detected in the 2D simulation due to the symmetric vortex structures. In general, the 2D model captures the main feature of the flow structure and the hydrodynamic forces captured from the 2D and 3D models match each other well.

\section{$3.2 K C=2$}

Strong 3D features are observed in the 3D simulation with $\mathrm{KC}=2$ and $\mathrm{Re}=2000$. Figure 9 shows the vorticity iso-surfaces of $\omega_{x}= \pm 2, \omega_{y}= \pm 2$ and $\omega_{z}= \pm 2$ at $K C=2$ at $t / T=20.25(\phi=$ $0.5 \pi)$. In Figure $9(\mathrm{a})$ and $(\mathrm{b})$, rib-like vortices $\left(\omega_{x}\right.$ and $\left.\omega_{y}\right)$ are found around the cylinder. The amplitudes of $\omega_{x}, \omega_{y}$ and $\omega_{z}$ are 54, 30 and 201 respectively. The strong vorticity in the $x$ - and $y$-directions $\left(\omega_{x}, \omega_{y}\right)$ causes the distortion of the spanwise vortices (Figure 9 (c)), where the span-wise vortices show strong chaotic features. The relative amplitudes of the three vorticity components and the chaotic vortex structures indicate that the flow is in the turbulent flow regime. This is in agreement with the experimental observation by Sarpkaya (1986). According to Sarpkaya (1986), the critical $\beta$ number for turbulent flow is about 600 at $K C=2$.

The middle section flow structures are examined and the vorticity $\left(\omega_{z}\right)$ contours are plotted in Figure 10 (a). It is observed that large amount of small scale irregular vortices exist around the main vortices next to cylinder surface. The small-scale vortices are induced by the strong vorticity in the $x$ - and $y$-axis directions. The boundary layer shown in Figure 10 (a) is smooth and attached to the cylinder surface. Figure 10 (b) shows the 2D simulation result, which is strictly symmetric with respect to the plane of $y=0$, corresponding to zero lift force. The major vortex structures shown in Figure 10 (a) and (b) resemble each other qualitatively. 
The variation of $C_{x}$ along the spanwise direction is very small, although the flow shows strong $3 \mathrm{D}$ features. This is mainly because $C_{x}$ is still dominated by the inertia component at $K C$ $=2$. The span-averaged $C_{x}$ is compared with $2 \mathrm{D}$ simulation results as shown in Figure 11 (a) and only very minor difference can be seen. The small-scale vortices shown in Figure 11 (a) induce low-amplitude sectional lift force. The lift forces in the section of $z / D=0.0$ and 2.0 and the span-averaged lift force are plotted in Figure 11 (b). The amplitude of sectional lift forces is about 0.2 and that of the span-averaged lift is about 0.05 . The amplitude of the sectional lift force is still two orders lower than that of the inline force. The 2D model gives zero lift as the flow structures is symmetric with respect to the $x$-axis.

\section{3 $K C=5$}

$\mathrm{KC}=5$ is in the transition from a non-shedding flow pattern (two attached vortices around the cylinder) to one-pair shedding regime (Williamson 1985). Lam and Dai (2002) investigated the formation of a vortex street at relatively low $\mathrm{KC}$ conditions $(\mathrm{KC}=4.25$ and $6.28, \mathrm{Re}=$ 5500 and 5900, respectively). Lam and Dai (2002) reported that the flow was mainly symmetric at $\mathrm{KC}=4.25$ and the flow contains two modes at $\mathrm{KC}=6.28$, switching between symmetric and asymmetric patterns. There has been much study about the flow structure visualization in a plane perpendicular to the cylinder, but very little information has been reported about the spanwise flow structure. It is expected that the present work will improve the understanding about the spanwise flow structure at this flow regime.

Figure 12 shows the vorticity iso-surfaces of $\omega_{x}= \pm 2, \omega_{y}= \pm 2$ and $\omega_{z}= \pm 2$ for $K C=5$ at the instant of $t / T=20.25(\phi=0.5 \pi)$. Strong chaotic vortex structures are observed. The intensity of vorticity in the three directions is at the same level. The vortices are obviously asymmetric with respect to the x-axis. The vortices are swept around the cylinder by the oscillatory flow but no vortex shedding happens. These features are also found in the 2D results, except for the small-scale vortices due to three-dimensionality, as shown in Figure 13. The autocorrelation of $\mathrm{u}$ velocity component $\left(R_{u u}\right)$ along the line of $x=0$ and $y / D=0.55$ was calculated over 10 flow period and plotted in Figure 14. It can be seen the correlation value decreased to zero at about $z / D=3.5$, which demonstrated that the span length of $8 \mathrm{D}$ is sufficient for the simulation.

The inline force shows strong periodicity and weak variation along the spanwise direction. The sectional $(z=0)$, span-averaged and the 2D inline forces are plotted in Figure 15 (a). The $C_{x}$ at section $\mathrm{z}=0$ and the span-averaged $C_{x}$ have minor difference, while the amplitude of $C_{x}$ from $2 \mathrm{D}$ modelling are about $10 \%$ higher than that of the $3 \mathrm{D}$ results. 
The asymmetric vortices on the top and bottom sides of the cylinder (Figure 13) lead to non-zero lift force. The 2D lift force is plotted in Figure 15 (b), which shows a strong secondary oscillation, with a secondary period of $25 T$. The secondary oscillation behaviour is induced by the un-coupling of vortex forming around the cylinder and flow oscillation, which has been reported by Dütsch et al., 1998, Uzunoglu et al. 2001, Elston et al. 2006 and Duclercq et al. 2011. The secondary oscillation corresponds to a regular switching of the vortices from a spatial configuration to its mirror image with respect to the $x$-axis. This phenomenon is especially obvious for low Re number condition. A detailed study about the quasi-periodic vortex shedding at $K C=4.7$ and $R e=188$ (Elston et al. 2006). The 3D model also captures certain quasi-periodic feature in the lift force time history. The sectional lift force $(z=0)$ from the 3D model is plotted in Figure 15 (c). It shows irregular undulations and intermittent switching behaviour, rather than oscillation with a regular secondary period. This is mainly due to the strong three-dimensionality of the oscillatory flow as shown in Figure 12. The spanaveraged lift (Figure 15 (c)) shows similar features but with a lower amplitude, indicating strong variation of lift along the spanwise direction.

The intermittent switching behaviour of the sectional lift force shown in Figure 15 (c) is closely related to the vortex structure development around the cylinder. To examine this behaviour, a zoom-in view of the lift force in the middle section is plotted in Figure 16. It is found the lift forces in different periods are different from each other. For example, at $t / T=$ $18.375,19.375$ and 20.375 , the lift corresponds to the troughs, while at $t / T=21.375,22.375$ and 23.375 , the lift corresponds to the peaks, at $t / T=24.375$, the lift force crosses zero and then switches back to troughs again at $t / T=25.375$ and 26.375. The flow structures corresponding to $\mathrm{t} / \mathrm{T}=19.375$ and 22.375 are presented in Figure 17. It is observed from Figure 17 (a) that a part of the negative vortices from the top of the cylinder just shed from the cylinder and a positive vortex from the lower side still attaches to the cylinder. Consequently a negative lift force was induced at this instance as shown in Figure 16. After three flow periods, the vortices around the cylinder switch to a different pattern as show in Figure 17 (b). This flapping behaviour of vortex structures corresponded to the switching of the lift force direction shown in Figure 16.

The evolution process of the distribution of lift force along the spanwise direction of the cylinder is presented in Figure 18. 3D flow structures emerge in the $9^{\text {th }}$ period and the threedimensionality increases gradually. After the $11^{\text {th }}$ period, strong variation of lift force along spanwise direction is found. The strong three-dimensionality of flow is clearly evident in Figure 12. Figure 18 show the lift force varies significantly along spanwise direction. The lift 
forces at two different $\mathrm{z}$ locations are in opposite signs sometimes. Consequently the spanwise averaged lift force has a much lower amplitude compared with the $2 \mathrm{D}$ simulation results. Clearly the 2D model is not suitable to simulate this flow condition due to the strong 3D nature of the flow at $K C=5$.

\section{$3.4 K C=10$}

The case of $K C=10$ investigated in the present study corresponds to a flow regime with a transverse vortex street described by Williamson (1985). In this flow regime, two vortices are shed from the same side of the cylinder and convected away from the cylinder in the transverse direction in each period of oscillatory flow. Duclercq et al. 2011 presented a series of numerical simulation results at $\mathrm{KC}=10 \mathrm{Re}=40 \sim 500$ and the research work was focused on the characterization of long time fluctuations of hydrodynamic force. It was noticed that the force and flow structures are very sensitive to Re number.

Figure 19 compares flow structures from the $2 \mathrm{D}$ and the 3D models at $t / T=15.25(\phi=$ $0.5 \pi)$. The major vortices are shed from the top side of the cylinder. Some small scale vortices exist under the cylinder in the 3D model results. The vortex shedding pattern is stable after the oscillatory flow is fully developed in both the 2D and 3D model results. Figure 19 (a) shows one negative vortices attaches on the top side of the cylinder (labelled as A) and a positive one attaches from the bottom side. The two vortices are developed in different stage with vortex A about to shed from the cylinder. The unbalanced vortices explained the pressure difference between the top and the bottom surface of the cylinder as shown in Figure 4. Vortex B is still fully attached to the cylinder surface and leads to a much stronger negative pressure zone on the bottom side of the cylinder surface.

To quantify the difference of the vorticity field, the 2D and 3D results about vorticity line with $\omega_{z}=-11$ to -15 on the top side of the cylinder are plotted together in Figure 20 (a). The 2D and 3D model predict the sepration point at the same location. The contour lines from the 2D and 3D model overlap with each other on the upstream surface of the cylinder before reaching the separation point. After separation point, there is an obvious difference. The vortex core from the 3D model in Figure 20 (a) is further away from the cylinder. This demonstrated that the $2 \mathrm{D}$ assumption lead to inaccurate prediction about the detailed flow structures separated from the cylinder, such as vortex core positions. The peak value of spanwise vorticity $\left(\omega_{z_{-} \max }\right)$ is also examined for this case as plotted in Figure 20 (b). It shows that $\left(\omega_{z_{-} \max }\right)$ is in the range of 55 to 160 . The fluctuation of $\omega_{z_{-} \max }$ is in phase with the free field velocity. The peak vorticity simulated using the $2 \mathrm{D}$ and $3 \mathrm{D}$ model agree with each other very well. This is mainly 
because the peak of vorticity always happens in the upstream boundary layer on the cylinder surface, where relatively low three-dimensionality is observed.

Figure 21 shows the sectional $(z=0)$, span-averaged and 2D results of $C_{x}$ and $C_{y}$, together with the experimental data from Obasaju et al. (1988). It is found that $C_{x}$ and $C_{y}$ in two succeeding half-periods have different peak values. This is because the two vortices that are shed in one period have different strengths. The 2D and 3D models give nearly identical hydrodynamic force results. There is an obvious difference between present numerical predicted drag force and the experimental data (Figure 21 (a)), but the lift force shows very good agreement with the experiment as shown in Figure 21 (b). The horizontal force on the cylinder was strongly dependent on the pressure difference between upstream and downstream of the cylinder, while the lift force was mainly governed by the pressure difference between the top and the bottom of the cylinder. The comparison shown in Figure 21 indicates that the numerical models capture the pressure difference between the top and the bottom of the cylinder better than the upstream-downstream pressure different in this particular flow condition. However this point could not be examined directly as no hydrodynamic pressure information in Obasaju et al. (1988). This discrepancy was also partially attributed to the Reynolds effect, although the Re number of this case was not given in Obasaju et al. (1988).

Figure 22 shows the evolution process of the lift force along the spanwise direction of the cylinder. The variation of the lift force along the spanwise direction in Figure 22 are much weaker than that at $\mathrm{KC}=5$ shown in Figure 18. Obasaju et al. (1988) measured the correlation coefficient of lift force on a circular cylinder in oscillatory flow. A correlation coefficient close to 1.0 was found at $K C=10$, indicating weak $3 \mathrm{D}$ effect. The distribution of lift force shown in Figure 22 agrees with the experimental finding of Obasaju et al. (1988). Obasaju et al. (1988) also reported that the vortex shedding pattern could transit to its mirror-image pattern only when the flow was restarted or strong perturbation was applied.

\section{$3.5 K C=17.5$}

The flow condition under the range of $16<K C<24$ was classified as double-pair regime (Williamson 1985 and Obasaju et al., 1988). In this flow regime, two pairs of vortices are shed from the cylinder and convected away from the cylinder in the diagonal directions. Figure 23 shows a comparison of $\omega_{z}$ between the results from the 2D and 3D models. Once again the 2D model captures the main feature of the flow structure well. The inline and lift results predicted by the two models also compares well as shown in Figure 24. The sectional lift force has 
strong correlation along the spanwise direction. It is also observed in Figure 24 that the lift force in the first and second half of flow period is asymmetric. This is mainly because of the vortices mainly convect away in the diagonal direction. As shown in Figure 23, in the second half of a flow period, the two vortices shed from the cylinder form a vortex pair and move to the top left direction. The vortex pair leads to a local pressure reduction on the top side of the cylinder and consequently a strong positive peak was observed at $t / T=0.75$. In the next half of the flow period, another vortex pair will move to the bottom right direction and cause a strong peak in the negative y-direction.

\section{$3.6 K C=20$}

$K C=20$ is found to be close to the critical $K C$ number between double-pair and three-pair vortex shedding regimes (Williamson 1985). Present numerical models show that the flow changes between the two-pair and three-pair vortex shedding regime intermittently, but it is dominated by the two-pair regime in most of the time. The frequency of lift force increases with the number of vortices shed from the cylinder in each flow period. The features described above are captured by both the 2D and 3D models.

The vortex shedding at two different spanwise locations can happen in different phase angle, although they are both in the two-pair vortex shedding regime. Figure 26 shows the time histories of inline and lift forces at two sections $(z / D=4$ and -4$)$ in five flow periods. It can be seen clearly that the lift forces at the two locations are in opposite directions. The opposite lift forces occur because the vortex shedding flow at $z / D=4$ is in anti-phase with that at $z / D=-4$. The inline forces at the two locations are slightly different from each other. The anti-phase vortex shedding flows at two locations can only be captured by $3 \mathrm{D}$ numerical modelling. Another phase change phenomenon is that after certain period of time, vortex shedding flow may changes to its anti-phase pattern. This shift in the vortex shedding pattern temporally is found both in the 2D and 3D numerical results.

The evolution of lift force along the spanwise direction is shown in Figure 27. The variation of lift force along spanwise direction is shown clearly in all the 20 flow periods plotted. The figure shows the unit-phase vortex shedding phenomenon mentioned in previous paragraph happens at different locations along the cylinder at different time. The timing of switching between different vortex shedding modes could be affected by the periodic boundary condition applied at the two ends of the cylinder, due to a limited length of 19.6D used in the simulation. It is worthwhile checking this point when further developed computational facility is available. 
The time histories of inline and lift forces calculated using the 2D model are plotted in Figure 28, in which, mode 1 stands for the two-pair flow regime, mode 2 is the anti-phase (mirror image) pattern of mode 1. Mode 3 stands for the three-pair regime. Obasaju et al. (1988) demonstrated that the three modes co-exist physically at this $\mathrm{KC}$ number. Due to the coexisting of the three flow modes, the mode-average method developed by Obasaju et al. (1988) is used here to separate the flow modes from each other. In this method, the flow periods with the same vortex shedding mode are summarized together for calculating the averaged hydrodynamic force. The mode-averaged forces are compared with each other in Figure 29. The $2 \mathrm{D}$ and $3 \mathrm{D}$ results of the mode-averaged force coefficient generally agree with each other (Figure 29 (a), (b) and (c)). The span-averaged results depicted in Figure 29 (d) shows that the amplitude is much lower than the mode-averaged results. This is because the different flow regimes along the spanwise directions are averaged, resulting averaged forces smaller than the sectional-forces. It is speculated the mirror image pattern of Mode 3 also exists physically, although it is neither captured in present numerical simulation, nor reported in the physical experiments by Obasaju et al. (1988).

\section{$3.7 K C=26.2$}

The results of $K C=26.2$ are presented here as a typical case in the three-pair vortex shedding regime. The flow structure along the spanwise direction shows a relatively strong correlation. Three pairs of vortices are shed from the cylinder in each flow period and mainly propagate along the flow oscillating direction. Detailed description of flow structure in this regime has been given in Williamson (1985), Obasaju et al. (1988) and Lam et al (2010). No numerical simulation data about flow structure is given here. The hydrodynamic forces predicted by the two models show good agreement as shown in Figure 30. The time history of hydrodynamic forces from the experimental work published by Obasaju et al. (1988) are also plotted as a reference and the numerical results match the experimental data well.

\subsection{Force coefficients}

In the above sections, the flow structures and force time histories calculated using the $2 \mathrm{D}$ model are compared with the results using the $3 \mathrm{D}$ model. In this section, the Morison coefficients derived from the 2D and 3D simulations are compared quantitatively. The drag coefficient $\left(C_{D}\right)$ and inertia coefficient $\left(C_{M}\right)$ are calculated based on the Morison equation as:

$$
F_{x}=\frac{1}{4} \rho \pi D^{2} C_{M} \frac{d u(t)}{d t}+\frac{1}{2} \rho D C_{D}|u(t)| u(t)
$$

The least-square method is used to calculate the values of $C_{D}$ and $C_{M}$. The predicted force coefficients from the numerical models are compared with published experimental data first. It 
has been known the force coefficients are sensitive to both $\mathrm{Re}$ and $\mathrm{KC}$. Therefore it is important to match both $\mathrm{Re}$ and $\mathrm{KC}$ between the numerical and experimental conditions to achieve a valid comparison. A comparison of Morison coefficients between the 2D, 3D numerical model and experimental data is given in Figure 31. The corresponding experimental flow conditions were $(\mathrm{KC}, \mathrm{Re})=(1,2070),(2,1380),(5,2415),(10,1960),(17.5,1907.5),(20$, 2180), (26.2, 2855.8). KC = 1 and 2 are selected from Sarpkaya (1986) and the other five KC numbers are from Obasaju et al. (1988). These conditions were selected because they are the closest to the numerical simulation conditions among the available published experimental data. It can be seen both the $C_{D}$ and $C_{M}$ values follow the trend observed in the experimental data closely. For $K C \geq 10$, the $C_{D}$ values from present work are slightly lower than the referred experimental data and the $C_{M}$ values agree with the experimental data well. In most of the case, the 2D and 3D model give very similar force coefficients. When there is an obvious different between $2 \mathrm{D}$ and $3 \mathrm{D}$ numerical results $(K C=5)$, the force coefficients from the 3D model are in better agreement with the experiments.

The 2D and 3D model results about drag and inertia coefficient are listed in Table 2 together the maximum and root-mean-square values of lift force $\left(C_{y \max }\right.$ and $\left.C_{y r m s}\right)$. The difference between of force coefficients from the 2D and 3D models are further analysed. The difference between the $2 \mathrm{D}$ and $3 \mathrm{D}$ results of $C_{M}$ is defined as

$\delta=\frac{C_{M, 2 D}-C_{M, 3 D}}{C_{M, 3 D}}$

The difference of other coefficients is defined in the same way. For $K C=1$ and 2, the inertia coefficients are predicted very well by the $2 \mathrm{D}$ model. The relative difference is just $0.2 \%$ and $2 \%$, respectively. At low $K C$ numbers, the inline force is dominated by the inertia force component, so the inline forces calculated using the 2D and 3D models agree with each other very well. However the drag coefficients predicted by the 2D model shows obvious difference from the $3 \mathrm{D}$ results ( $\delta=8.79 \%$ and $12.95 \%$, respectively) at $\mathrm{KC}=1$ and 2 , indicating the three-dimensionality of the flow has significant effect on the drag forces acting on the cylinder. The 3D flow around the cylinder leads to oscillatory lift forces at $K C=1$ and 2, however the amplitudes are negligibly smaller than that of the corresponding inline force.

The strongest three-dimensionality of oscillatory flow occurs at $K C=5$. The $2 \mathrm{D}$ model is not able to predict the force coefficients accurately. As shown in Table 1, the 2D model predicts the $C_{\mathrm{M}}, C_{\mathrm{D}}, C_{\mathrm{ymax}}$ and $C_{\mathrm{yrms}}$ with very big differences of $8.91 \%, 19.97 \%, 96.64 \%$ and 
$163.54 \%$, respectively. Reasonable good agreement is found between 2D and 3D results of force coefficients of cases of $K C=10,17.5,20$ and 26.2.

\section{Conclusions}

Oscillating flow around a circular is investigated by solving the 2D and 3D Navier-Stokes equations using the PG-FEM method. Calculations are carried out for a constant Reynolds number of 2000 and seven $K C$ numbers of 1, 2, 5, 10, 17.5, 20 and 26.2. The conclusions are summarized as follows.

The flow at $K C=1$ is in the laminar flow regime, although 3D flow is observed around the cylinder. The major features of sectional vortex structures are captured by the $2 \mathrm{D}$ model. The 3D flow structures induce oscillatory lift, but the amplitude of the lift is low. The inline force is predicted by the $2 \mathrm{D}$ model with high accuracy.

The flow of $K C=2$ is in the turbulent flow regime. Small-scale vortices are found in sectional flow structures. The vortex structures simulated by the 2D model match the major sectional vortex structures from the 3D model. The lift force is still negligibly small and the inline force is well predicted by the 2D model.

The flow of $K C=5$ shows the strongest three-dimensionality as shown in the 3D simulation results. The vortices on the two sides of the cylinder are developed with different strength, resulting in a non-zero lift force. The sectional lift forces show intermittent switching behaviour, which is closely related to the vortex structure change around the cylinder. Strong variation of flow structures along the spanwise direction is observed. The regular secondary period observed in the 2D model does not exist in $3 \mathrm{D}$ simulation. The amplitude of the lift force predicted by the $2 \mathrm{D}$ model is much higher than that by the $3 \mathrm{D}$ model. The $2 \mathrm{D}$ model predicts the inline force reasonably well.

The flows at $K C=10,17.5$ and 26.2 are in the single-pair, two-pair and three-pair vortex shedding regimes, respectively. At these three $K C$ numbers, the sectional lift forces and spanwise vortex structures show strong correlation. The $2 \mathrm{D}$ flow structure calculated using the 2D model agrees with the sectional flow structure and the two dimensional model predicts the inline and lift force well.

$K C=20$ is the critical $K C$ number between two-pair regime and three-pair regime, where multiple vortex shedding modes coexist. The mode-average method is used to analyse the lift force. The 2D mode-averaged forces are in good agreement with the 3D mode-averaged results. 
The comparison between the $2 \mathrm{D}$ and $3 \mathrm{D}$ results of force coefficients shows that the $2 \mathrm{D}$ model tends to under-predict $C_{M}$ by maximally $9.71 \%$ at $K C=20$ and tends to over-predict the $C_{D}$ by maximally $17.97 \%$ at $K C=5$ for the cases simulated in present study. At low KC values ( 1 and 2 ), the flow structures simulated using the 2D model are symmetric and leads to zero lift force, but the flow structures are asymmetric with respect to the $\mathrm{x}$-axis and generated non zero lift force in 3D model. This the large discrepancy of lift coefficients predicted by the two models. For larger $K C$ values ( $\geq 10$ ), the difference between lift coefficients is much smaller.

There is a good agreement of peak vorticity modelled by the 2D and 3D model since the peak vorticity normally appear at the boundary layer on the cylinder surface before reaching the separation point. There is obvious difference in term of detailed vortex locations between the $2 \mathrm{D}$ and $3 \mathrm{D}$ model results.

\section{Acknowledgements}

The authors would like to acknowledge the supports from Australia Research Council through ARC Discovery Project (Grant No. DP110105171), ARC Linkage Projects Program (Grant No. LP0989936). The numerical simulations were carried out on the supercomputing facility provided by iVEC.

\section{Reference}

An, H., Cheng, L., and Zhao, M. 2011 Direct numerical simulation of oscillatory flow around a circular cylinder at low $K C$ number. Journal of Fluid Mechanics. 666, 77-103.

Brooks, A. N. and Hughes, T. J. R. 1982. Streaming upwind/Petrov-Galerkin formulations for convection dominated flow with particular emphasis on the incompressible Navier-Stokes equations. Computer Methods in Applied Mechanics and Engineering. 32: 199-259.

Duclercq, M., Broc, D. and Cadot, O. 2011. Characterization of long time fluctuations of forces exerted on an oscillating circular cylinder at $\mathrm{KC}=10$. Journal of Fluids and Structures. 27, 596-610.

Elston, J. R., Blackburn, H. M. and Sheridan, J. 2006. The primary and secondary instabilities of flow generated by an oscillating circular cylinder. Journal of Fluid Mechanics. 550, 359389.

Honji, H. 1981. Streaked flow around an oscillatory circular cylinder. Journal of Fluid Mechanics. 107, 509-520.

Justesen, P. 1991. A numerical study of oscillating flow around a circular cylinder. Journal of Fluid Mechanics. 222, 157-196. 
Lam, K. M. and Dai, G. Q. 2002. Formation of vortex street and vortex pair form a circular cylinder oscillating in water. Experimental Thermal and Fluid Science. 26, 601-915.

Lam, K. M., Hu, J. C. and Liu, P. 2010. Vortex formation processes from an oscillating circular cylinder at high Keulegan-Carpenter numbers. Physics of Fluids. 22, 015105.

Lin, X. W., Bearman, P. W. and Graham, J. M. R. 1996. A numerical study of oscillatory flow about a circular cylinder for low values of beta parameter. Journal of Fluids and Structures. 10 (5): 501-526.

Nehari, D., Armenio, V., and Ballio, F. 2004. Three-dimensional analysis of the unidirectional oscillatory flow around a circular cylinder at low Keulegan-Carpenter and b numbers, Journal of Fluid Mechanics. 520, 157-186.

Obasaju, E. D., Bearman, P. W. and Graham, J. M. R. 1988. A study of forces, circulation and vortex patterns around a circular cylinder in oscillatory flow. Journal of Fluid Mechanics. $196,476-494$.

Saghafian, M., Stansb, P. K. and Saidi, M. S. and Apsley, D. D. 2003. Simulation of turbulent flows around a circular cylinder using nonlinear eddy-viscosity modelling: steady and oscillatory ambient flows. Journal of Fluids and Structures. 17, 1213-1236.

Sarpkaya, T. 1986. Force on a circular cylinder in viscous oscillatory flow at low KeuleganCarpenter numbers. Journal of Fluid Mechanics. 165, $61-71$.

Sarpkaya, T. 2002. Experiments on the stability of sinusoidal flow over a circular cylinder. Journal of Fluid Mechanics. 457, 157-180.

Scandura, P., Armenio, V. and Foti, E. 2009. Numerical investigation of the oscillatory flow around a circular cylinder close to a wall at moderate Keulegan-Carpenter and low Reynolds numbers. Journal of Fluid Mechanics. 627, 2509-290.

Tatsuno, M. and Bearman, P. W. 1990. A visual study of the flow around an oscillating circular cylinder at low Keulegan-Carpenter numbers and low Stokes numbers. Journal of Fluid Mechanics. 211, 157-182.

Yang, K., Cheng, L., An, H., Bassom A. and Zhao, M. 2014. Effects of an axial flow component on the Honji instability. Journal of Fluids and Structures, 49, pp. 614-639

Wang, X. and Dalton, C. Oscillatory flow past a rigid circular cylinder: a finite difference calculation. Journal of Fluids Engineering. 1990; 113: 377 - 383.

Williamson, C. H. K. 1985. Sinusoidal flow relative to circular cylinders. Journal of Fluid Mechanics. 155, 141-174.

Zhang, J. and Dalton, C. 1999. The onset of three-dimensionality in an oscillating flow past a fixed circular cylinder. International Journal for Numerical Methods in Fluids. 30, 19-42. 
Zhao, M, Cheng, L. and Zhou, T. 2011. Three-dimensional numerical simulation of oscillatory flow around a circular cylinder at right and oblique attacks. Ocean Engineering, 38, (1718), 2056-2069 
Table 1. Parameters of mesh dependency study $\left(\boldsymbol{N}_{\text {cylinder }}\right.$ is nodes number on the cylinder surface, $\boldsymbol{N}_{\text {total }}$ is the total nodes number in the domain, $\boldsymbol{N}_{\text {span }}$ is the element number along the span of the cylinder, $\Delta_{\boldsymbol{Z}}$ is the element size along spanwise direction).

\begin{tabular}{|l|l|l|l|l|l|}
\hline Mesh No. & Dimensions & $N_{\text {cylinder }}$ & \multicolumn{1}{|c|}{$N_{\text {total }}$} & $N_{\text {span }}$ & $\Delta_{Z} / \mathrm{D}$ \\
\hline 1 & 2D & 60 & 4932 & - & - \\
\hline 2 & 2D & 80 & 6270 & - & - \\
\hline 3 & 2D & 100 & 7850 & - & - \\
\hline 4 & 3D & 80 & $0.62 \times 10^{6}$ & 99 & 0.2 \\
\hline 5 & 3D & 80 & $1.24 \times 10^{6}$ & 197 & 0.1 \\
\hline 6 & 3D & 80 & $2.46 \times 10^{6}$ & 393 & 0.05 \\
\hline
\end{tabular}

Table 2. The comparison of 2D and 3D results of force coefficients.

\begin{tabular}{|l|c|c|c|c|c|c|c|c|c|c|c|c|}
\hline \multirow{2}{*}{$K C$} & \multicolumn{4}{|c|}{$C_{M}$} & \multicolumn{4}{|c|}{$C_{D}$} & \multicolumn{3}{c|}{$C_{y \max }$} & \multicolumn{3}{c|}{$C_{y r m s}$} \\
\cline { 2 - 13 } & $2 \mathrm{D}$ & $3 \mathrm{D}$ & $\delta(\%)$ & $2 \mathrm{D}$ & $3 \mathrm{D}$ & $\delta(\%)$ & $2 \mathrm{D}$ & $3 \mathrm{D}$ & $\delta(\%)$ & $2 \mathrm{D}$ & $3 \mathrm{D}$ & $\delta(\%)$ \\
\hline 1 & 2.035 & 2.031 & 0.20 & 0.879 & 0.808 & 8.79 & 0 & 0 & 0 & 0 & 0 & 0 \\
\hline 2 & 2.035 & 2.078 & -2.07 & 0.811 & 0.718 & 12.95 & 0 & 0.10 & 100 & 0 & 0.03 & 100 \\
\hline 5 & 1.778 & 1.952 & -8.91 & 1.503 & 1.274 & 17.97 & 2.542 & 1.362 & 86.64 & 0.730 & 0.277 & 163.54 \\
\hline 10 & 1.078 & 1.098 & -1.82 & 2.193 & 2.235 & -1.88 & 2.656 & 3.069 & -13.46 & 1.335 & 1.582 & -15.61 \\
\hline 17.5 & 1.222 & 1.298 & -5.86 & 2.036 & 1.975 & 3.09 & 2.553 & 2.553 & 0.00 & 1.067 & 1.081 & -1.30 \\
\hline 20 & 1.255 & 1.390 & -9.71 & 1.830 & 1.809 & 1.16 & 1.857 & 1.970 & -5.74 & 0.712 & 0.821 & -13.28 \\
\hline 26.2 & 1.343 & 1.472 & -8.76 & 1.668 & 1.667 & 0.06 & 1.670 & 1.495 & 11.71 & 0.677 & 0.652 & 3.83 \\
\hline
\end{tabular}


Figure 1. The critical $K C$ and $\beta$ values for flow transition from $2 \mathrm{D}$ to $3 \mathrm{D}$

Figure 2. A definition sketch of the computational domain.

Figure 3. The 2D computational mesh around the cylinder.

Figure 4. The pressure distribution around the cylinder at peak velocity and $\mathrm{KC}=10$ for mesh dependency study.

Figure 5. The spanwise vorticity iso-surfaces $\left(\omega_{z}= \pm 5\right)$ at $K C=10$ and $t / T=10.25(\phi=0.5 \pi)$ (flow in positive $x$-direction).

Figure 6. The vorticity iso-surfaces of $\omega_{x}= \pm 0.5, \omega_{y}= \pm 0.5$ and $\omega_{z}= \pm 0.5$ at the instant of $t / T=30.25(\phi=$ $0.5 \pi)$. The streamlines in the plane of $z / D=-4.0$ are plotted in (c) to indicate the flow direction. $(K C=1)$.

Figure 7. The sectional and two-dimensional vorticity contours $\left(\omega_{z}\right)$ at $t / T=30.25(\phi=0.5 \pi, K C=1)$.

Figure 8 . The $C_{x}$ time history calculated by $2 \mathrm{D}$ and $3 \mathrm{D}$ models.

Figure 9. The vorticity iso-surfaces of $\omega_{x}= \pm 2, \omega_{y}= \pm 2$ and $\omega_{z}= \pm 2$ at the instant of $t / T=20.25(\phi=0.5 \pi)$. The streamlines in the plane of $z / D=-4.0$ are plotted in (c) to indicate the flow direction $(K C=2)$.

Figure 10. The vorticity contours calculated by $2 \mathrm{D}$ and $3 \mathrm{D}$ models $((\phi=0.5 \pi))$.

Figure 11. The $C_{x}$ and $C_{y}$ calculated by $2 \mathrm{D}$ and $3 \mathrm{D}$ models at $K C=2$.

Figure 12. The vorticity iso-surfaces of $\omega_{x}= \pm 2, \omega_{y}= \pm 2$ and $\omega_{z}= \pm 2$ at the instant of $t / T=20.25(\phi=0.5 \pi)$. The streamlines in the plane of $z / D=-4$ are plotted in (c) to indicate the flow direction $(K C=5)$.

Figure 13 . The vorticity contours $\left(\omega_{z}\right)$ at $K C=5$.

Figure 14. The autocorrelation of $\mathrm{u}$ along spanwise direction $(\mathrm{x}=0$ and $\mathrm{y} / \mathrm{D}=0.55)$ at $K C=5$.

Figure 15. A comparison of sectional, span-averaged and two-dimensional $C_{x}$ and $C_{y}$ at $K C=5$.

Figure 16. A zoom-in view of the sectional $C_{y}(3 \mathrm{D}, z=0)$ at $K C=5$.

Figure 17. The sectional vorticity contours $\omega_{z}$ in the plane of $z=0$ at $K C=5$.

Figure 18. The evolution process of the lift force along the spanwise direction at $K C=5$.

Figure 19. The vortex structures $\left(\omega_{z}\right)$ of $K C=10(t / T=15.25,(\phi=0.5 \pi))$.

Figure 20. A comparions of vorticity contours of $\mathrm{KC}=10,(\mathrm{a}), \omega_{z}=-11 \sim-15$ at $\mathrm{t} / \mathrm{T}=15.25(\phi=0.5 \pi),(\mathrm{b})$, $\omega_{z_{-} \max }$ in one flow period.

Figure 21. The comparison of the two-dimensional, sectional and span-averaged $C_{x}$ and $C_{y}$ at $K C=10$.

Figure 22. The evolution process of sectional $C_{y}$ along the spanwise direction at $K C=10$.

Figure 23. The vortex structures $\left(\omega_{z}\right)$ of $K C=17.5(t / T=15.75,(\phi=0.5 \pi))$.

Figure 24. A comparison between the two-dimensional, sectional and span-averaged $C_{x}$ and $C_{y}$ at $K C=17.5$.

Figure 25. The evolution process of sectional $C_{y}$ along the spanwise direction at $K C=17.5$.

Figure 26. The time histories of sectional forces of $K C=20(z / D=-4$ and 4$)$.

Figure 27. The evolution process of sectional $C_{y}$ along the spanwise direction at $K C=20$.

Figure 28. The time histories of $C_{x}$ and $C_{y}$ of $K C=20$ (2D simulation results). 
Figure 29. A comparison between the two-dimensional, sectional and span-averaged $C_{x}$ and $C_{y}$ at $K C=20$.

Figure 30. A comparison between the two-dimensional, sectional and span-averaged $C_{x}$ and $C_{y}$ at $K C=26.2$. Figure 31. A comparison of Morison coefficients between the 2D, 3D numerical model and experimental data (the corresponding experimental flow conditions $(K C, R e)=(1,2070),(2,1380),(5,2415),(10,1960),(17.5$, 1907.5), (20,2180), (26.2, 2855.8), $K C=1$ and 2 were from Sarpkaya (1986) and the other five $K C$ numbers are from Obasaju et al. (1988)) 


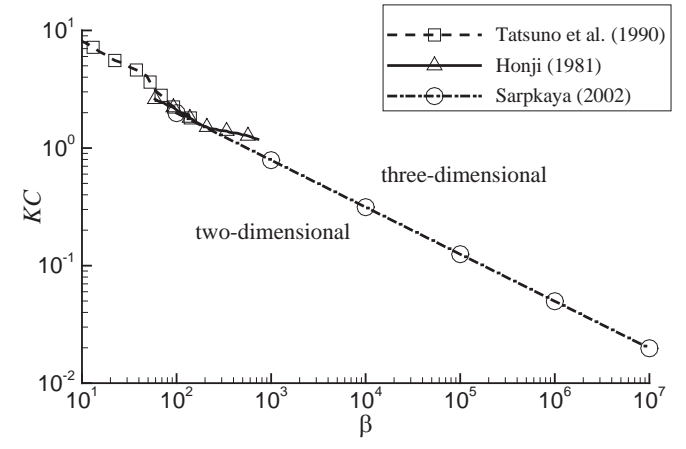

Figure 1. The critical $K C$ and $\beta$ values for flow transition from $2 \mathrm{D}$ to 3D

(a) $2 \mathrm{D}$

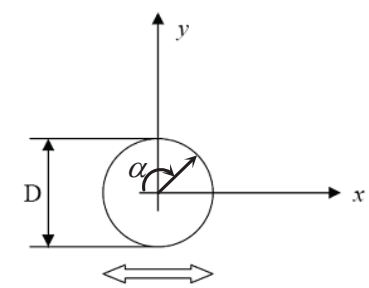

(b) $3 \mathrm{D}$

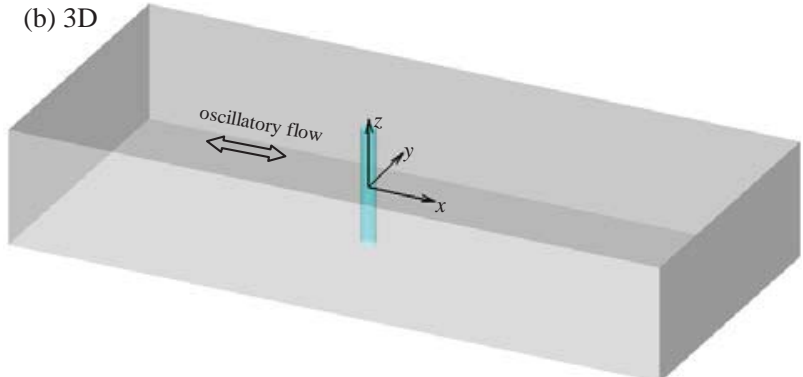

Figure 2. A definition sketch of the computational domain.

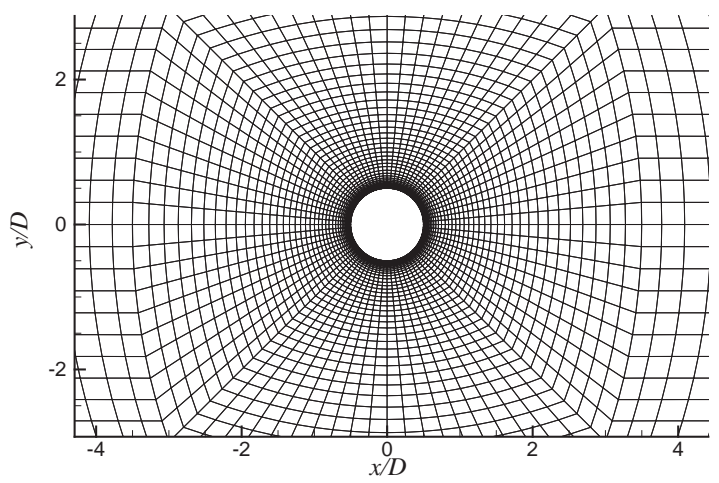

Figure 3. The 2D computational mesh around the cylinder. 

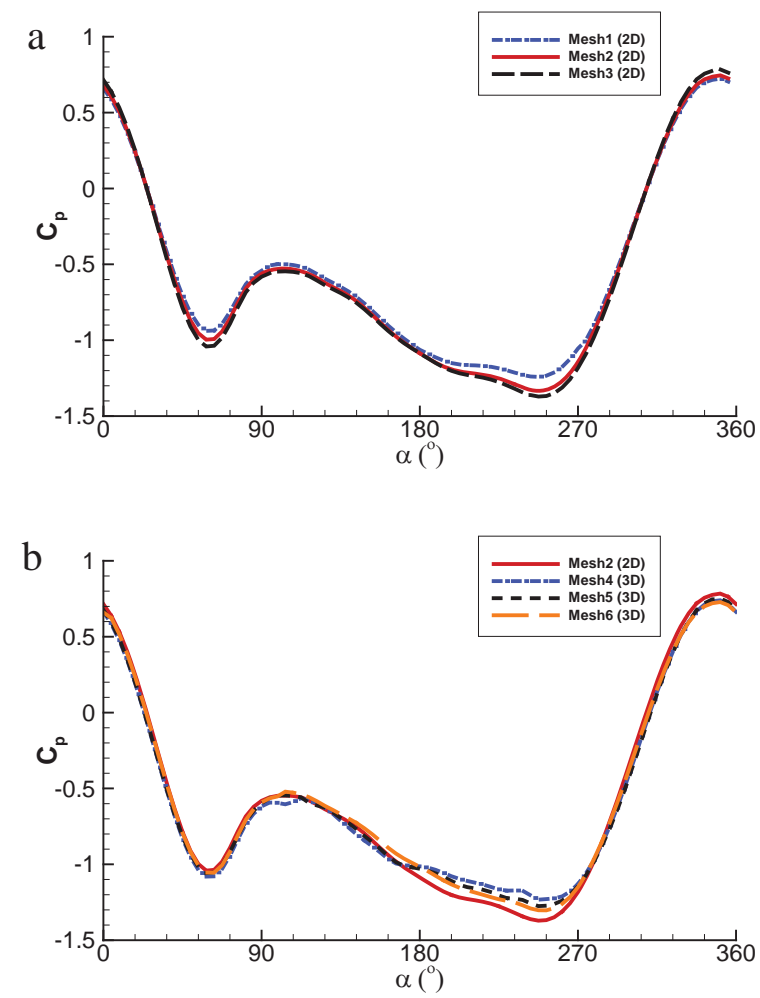

Figure 4. The pressure distribution around the cylinder at peak velocity and KC $=10$ for mesh dependency study, (a), two-dimensional mesh tests, (b), three-dimensional mesh tests.

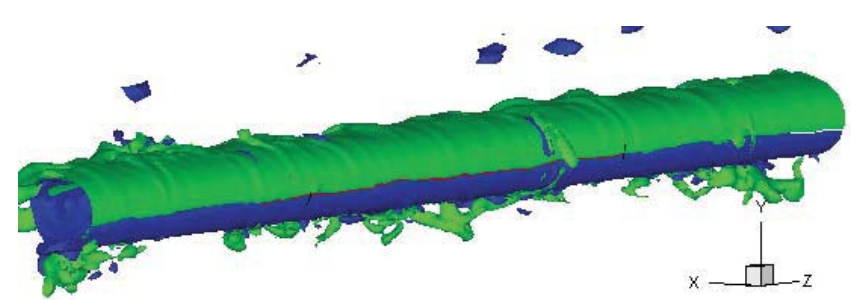

(a) Upstream view

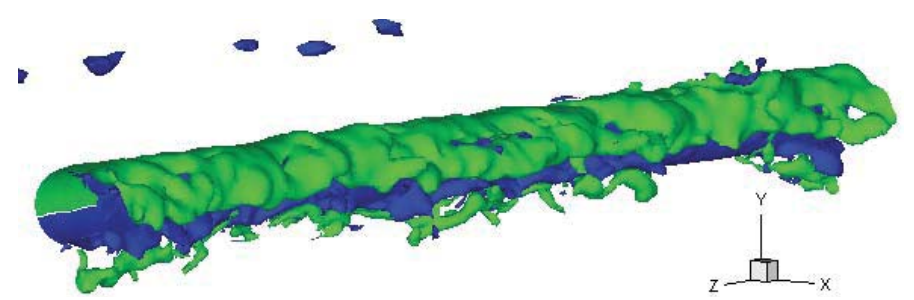

(b) Downstream view

Figure 5. The spanwise vorticity iso-surfaces ( $\omega_{z}= \pm 5$, green represents negative vorticity and blue represents positive vorticity) at $K C=10$ and $t / T=10.25$ (flow in positive $x$-direction). 
(a) $\omega_{x}$

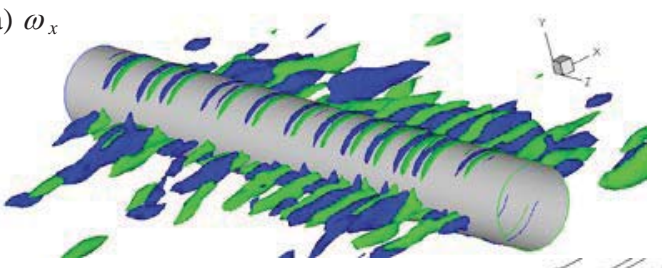

(c) $\omega_{z}$ (b) $\omega_{y}$

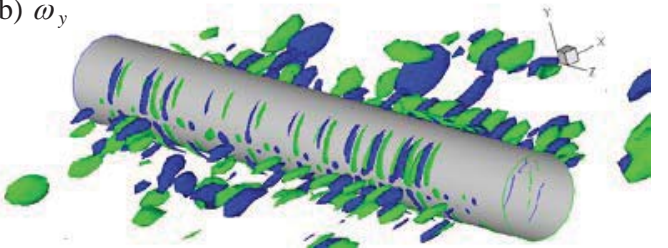

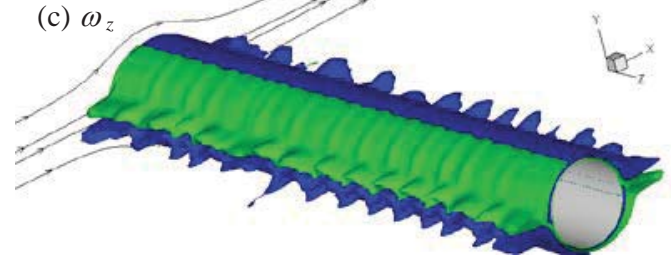

Figure 6. The vorticity iso-surfaces of $\omega_{x}= \pm 0.5, \omega_{y}= \pm 0.5$ and $\omega_{z}= \pm 0.5$ at the instant of $t / T=30.25$. The streamlines in the plane of $z / D=-4.0$ are plotted in (c) to indicate the flow direction. $(K C=1)$.
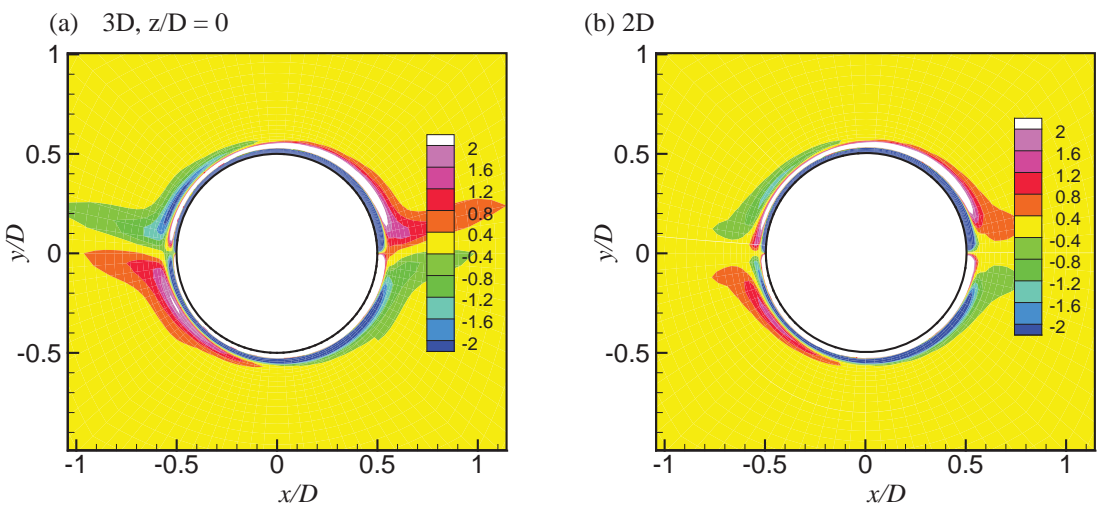

Figure 7. The sectional and two-dimensional vorticity contours $\left(\omega_{z}\right)$ at $t / T=30.25(K C=1)$.

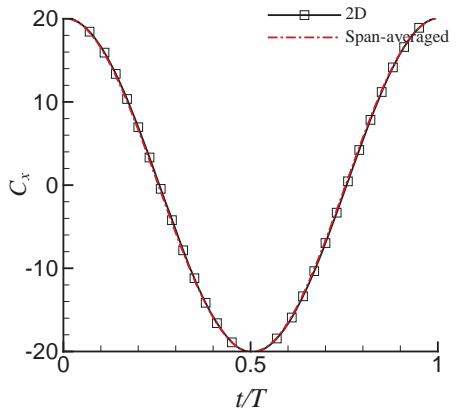

Figure 8 . The $C_{x}$ time history calculated by 2D and 3D models. 
(a) $\omega_{x}$

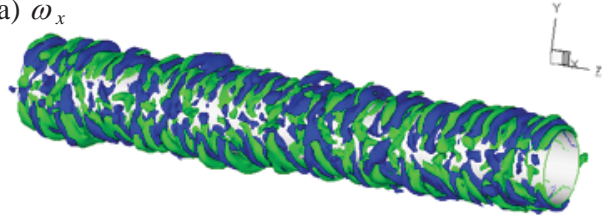

(b) $\omega_{y}$

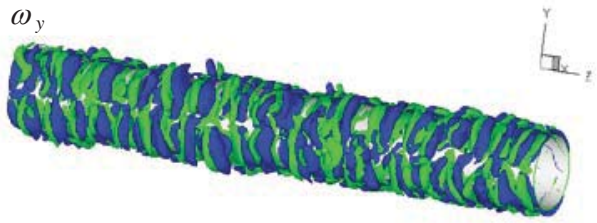

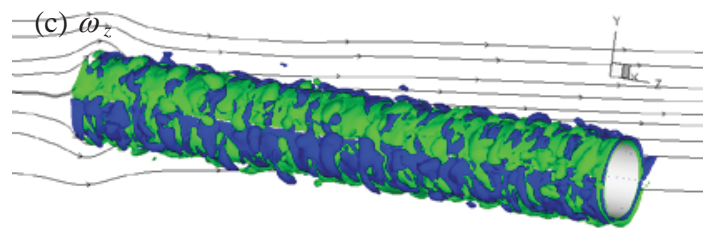

Figure 9. The vorticity iso-surfaces of $\omega_{x}= \pm 2, \omega_{y}= \pm 2$ and $\omega_{z}= \pm 2$ at the instant of $t / T=20.25$. The streamlines in the plane of $z / D=-4.0$ are plotted in (c) to indicate the flow direction $(K C=2)$.
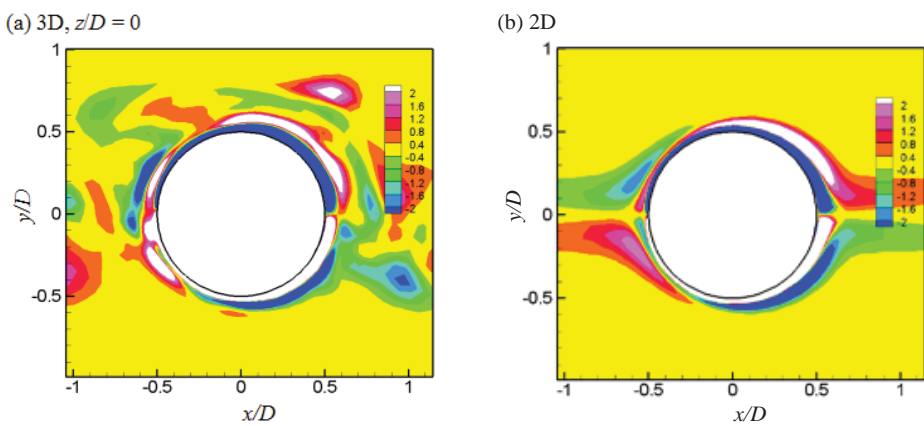

Figure 10 . The vorticity contours calculated by 2D and 3D models $(t / T=0.25)$.
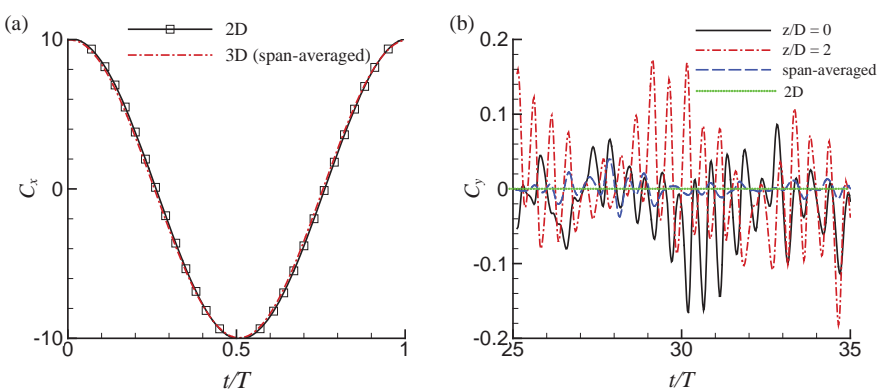

Figure 11. The $C_{x}$ and $C_{y}$ calculated by 2D and 3D models at $K C=2$. 


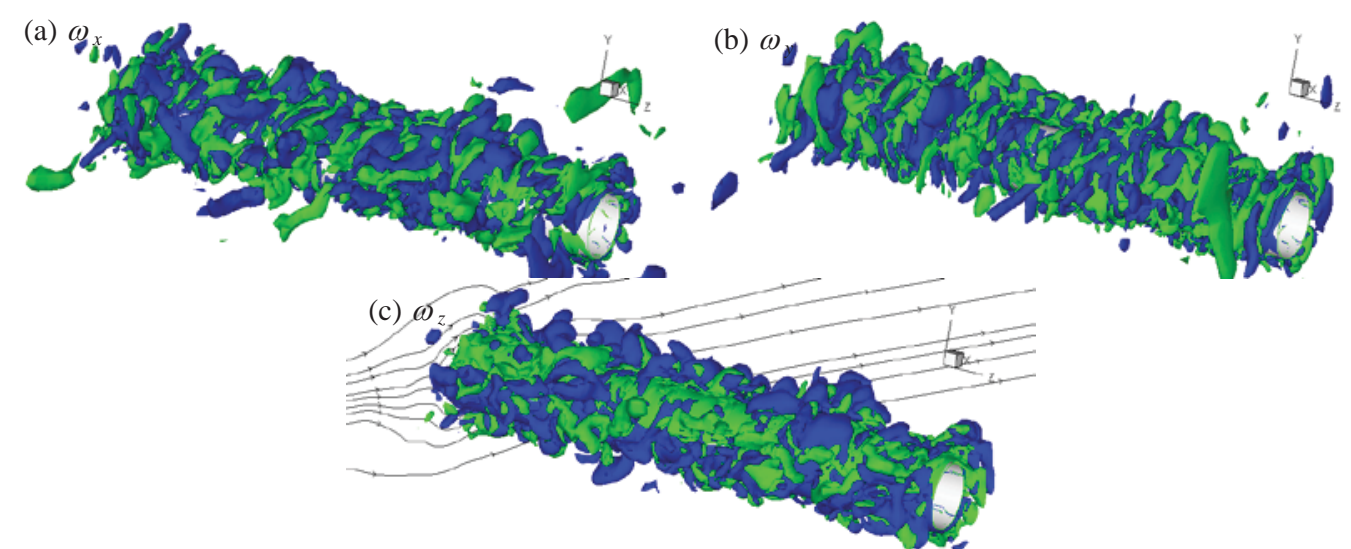

Figure 12. The vorticity iso-surfaces of $\omega_{x}= \pm 2, \omega_{y}= \pm 2$ and $\omega_{z}= \pm 2$ at the instant of $t / T=20.25$. The streamlines in the plane of $z / D=-4$ are plotted in (c) to indicate the flow direction $(K C=5)$.

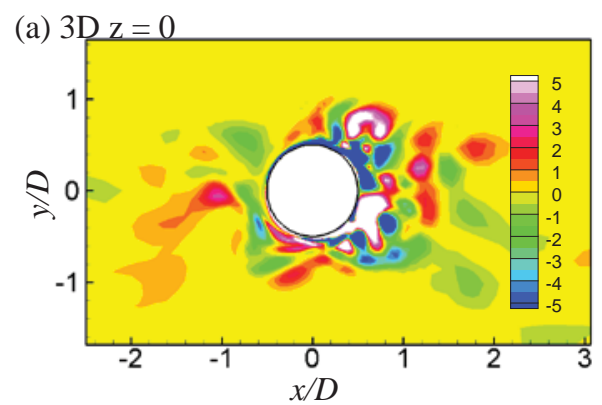

(b) $2 \mathrm{D}$

Figure 13 . The vorticity contours $\left(\omega_{z}\right)$ at $K C=5$.

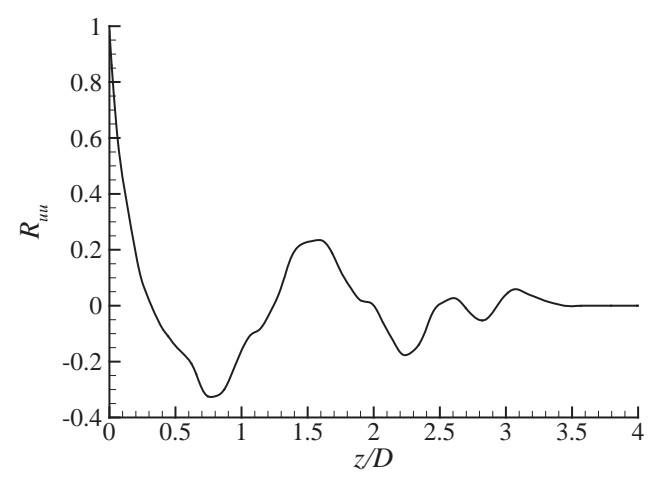

Figure 14. The autocorrelation of $\mathrm{u}$ along spanwise direction $(\mathrm{x}=0$ and $\mathrm{y} / \mathrm{D}=0.55)$ at $K C=5$.
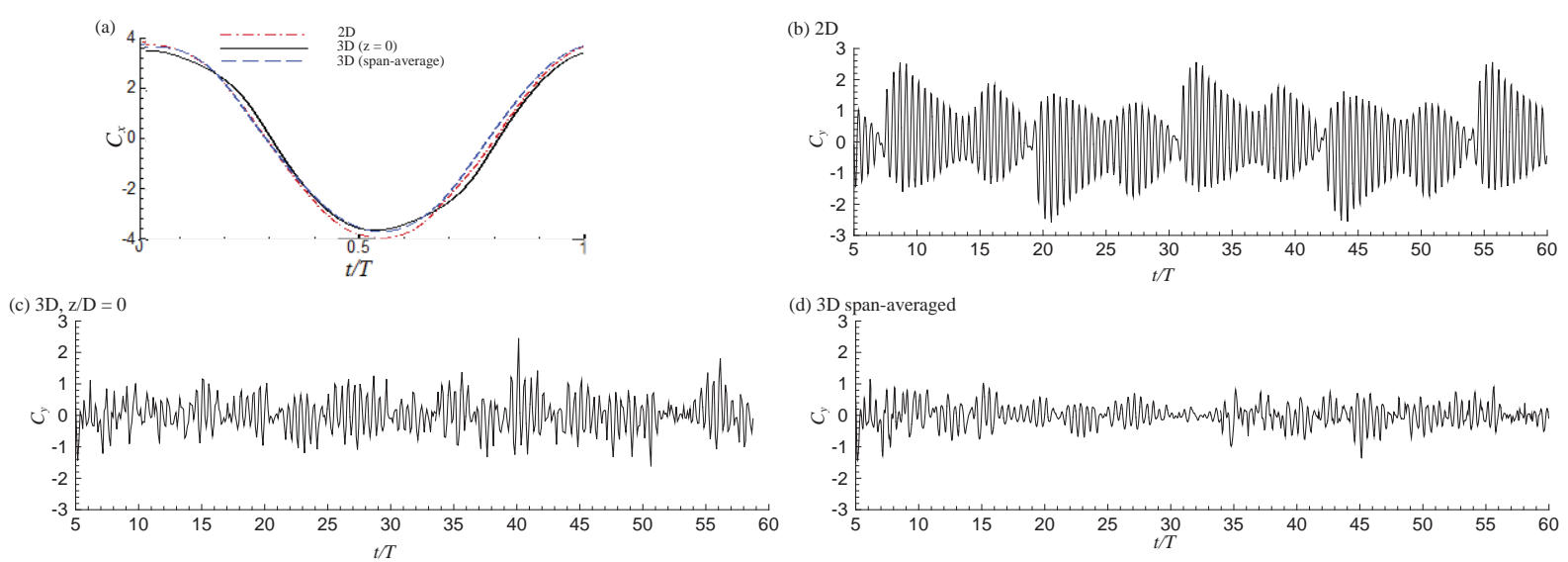
Figure 15. A comparison of sectional, span-averaged and two-dimensional $C_{x}$ and $C_{y}$ at $K C=5$.

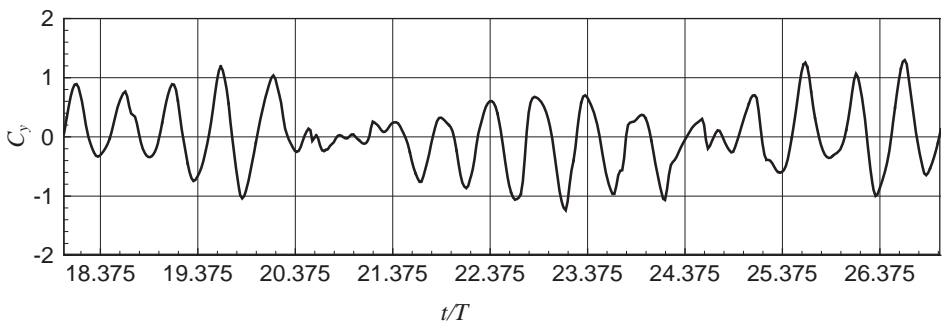

Figure 16. A zoom-in view of the sectional $C_{y}(3 \mathrm{D}, z=0)$ at $K C=5$.
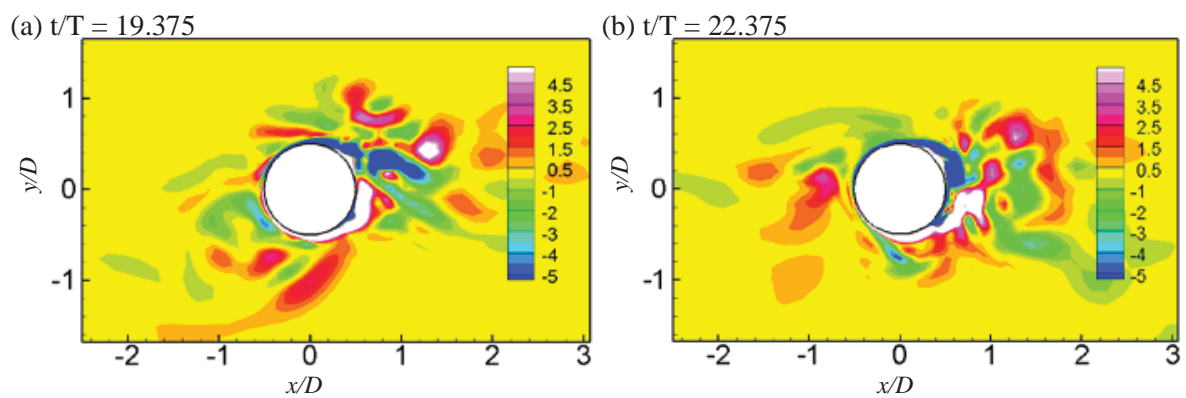

Figure 17. The sectional vorticity contours $\omega_{z}$ in the plane of $z=0$ at $K C=5$.

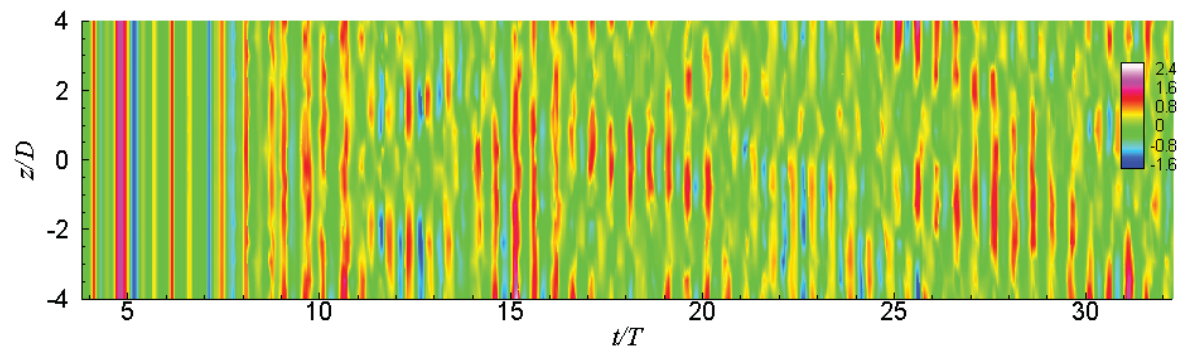

Figure 18. The evolution process of the lift force along the spanwise direction at $K C=5$.

(a) $2 \mathrm{D}$

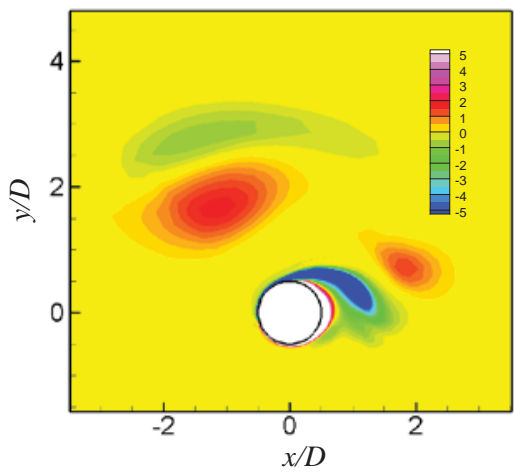

(b) $3 \mathrm{D}, \mathrm{z}=0$

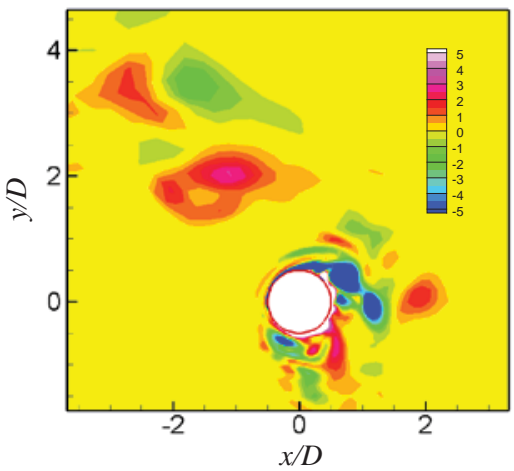

Figure 19. The vortex structures $\left(\omega_{z}\right)$ of $K C=10(t / T=15.25)$. 

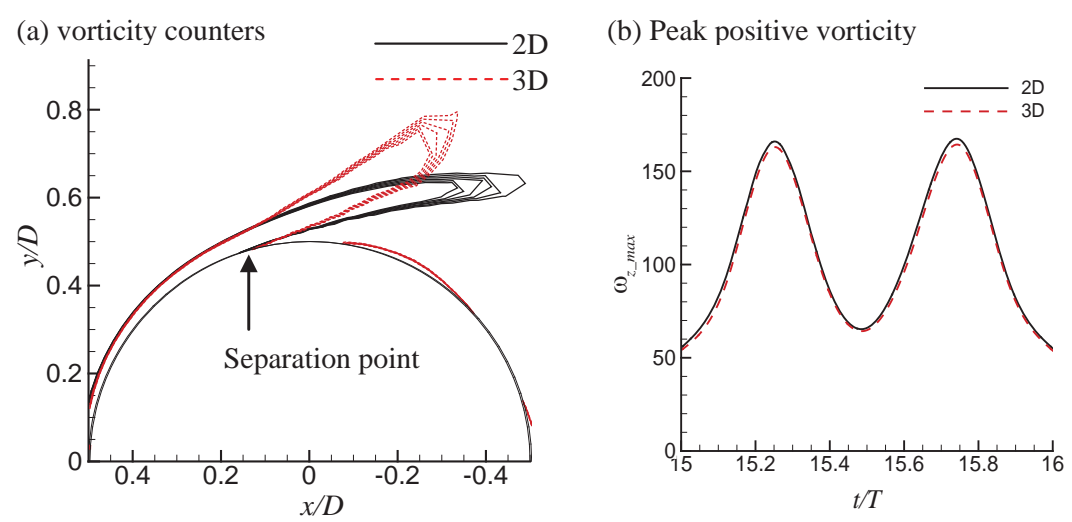

Figure 20. A comparions of vorticity contours of $\mathrm{KC}=10,(\mathrm{a}), \omega_{z}=-11 \sim-15$ at $\mathrm{t} / \mathrm{T}=15.25$, (b), $\omega_{z_{-}} \max$ in one flow period.
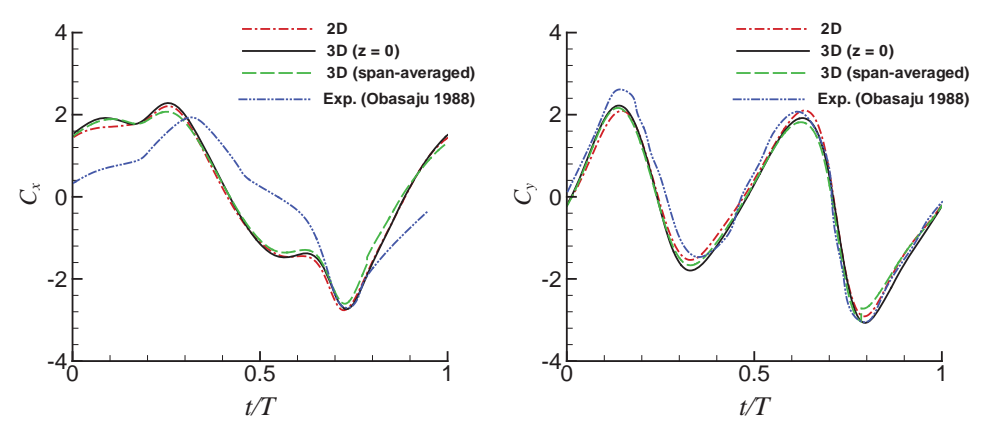

Figure 21. The comparison of the two-dimensional, sectional and span-averaged $C_{x}$ and $C_{y}$ at $K C=10$ together with the experimental data given by Obasaju et al. (1988).

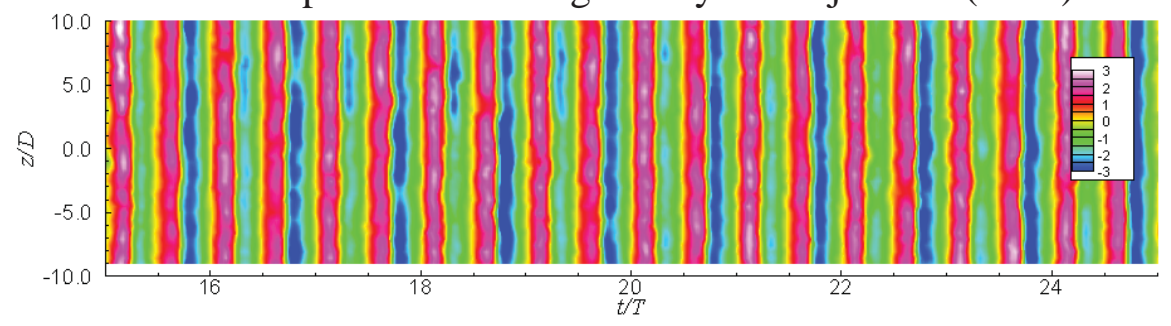

Figure 22. The evolution process of sectional $C_{y}$ along the spanwise direction at $K C=10$.

(a) $3 \mathrm{D}, \mathrm{z}=0$

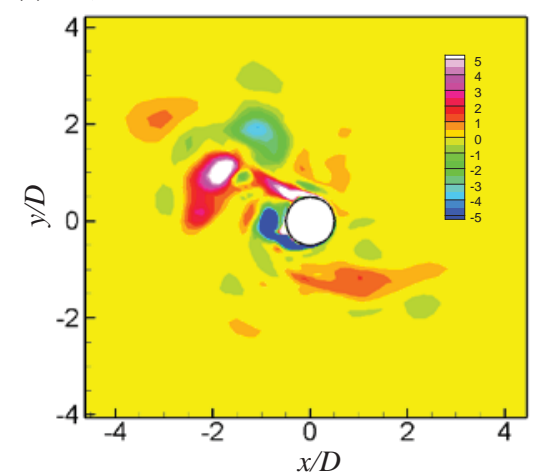

(b) $2 \mathrm{D}$

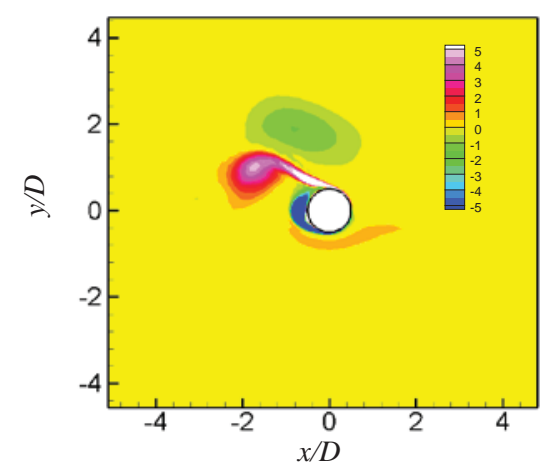

Figure 23. The vortex structures $\left(\omega_{z}\right)$ of $K C=17.5(t / T=15.75)$. 

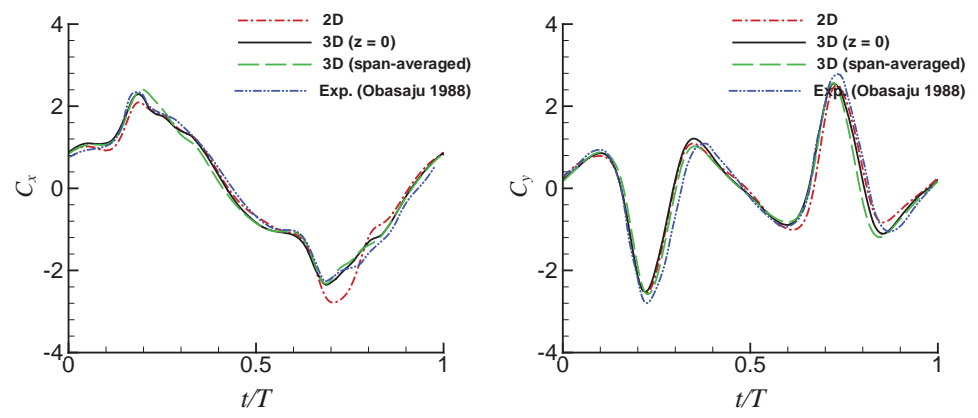

Figure 24. A comparison between the two-dimensional, sectional and span-averaged $C_{x}$ and $C_{y}$ at $K C=17.5$.

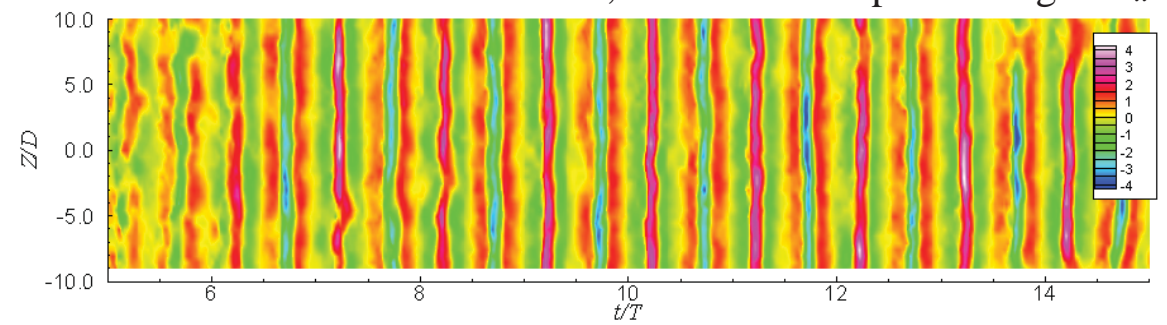

Figure 25. The evolution process of sectional $C_{y}$ along the spanwise direction at $K C=17.5$.
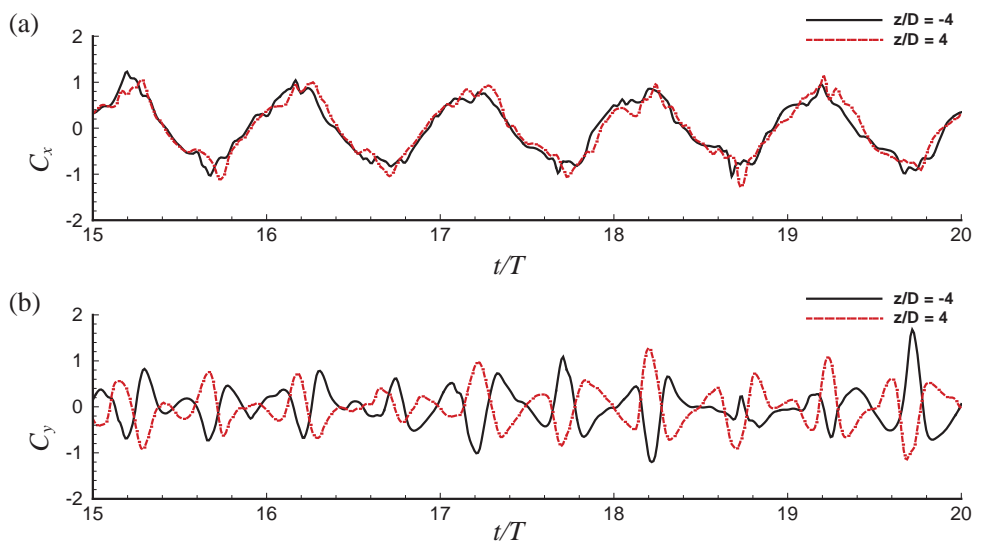

Figure 26. The time histories of sectional forces of $K C=20(z / D=-4$ and 4$)$.

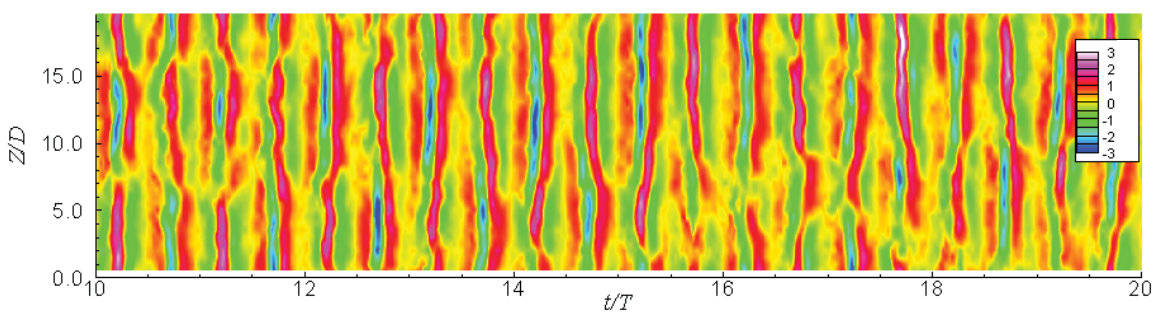

Figure 27. The evolution process of sectional $C_{y}$ along the spanwise direction at $K C=20$. 

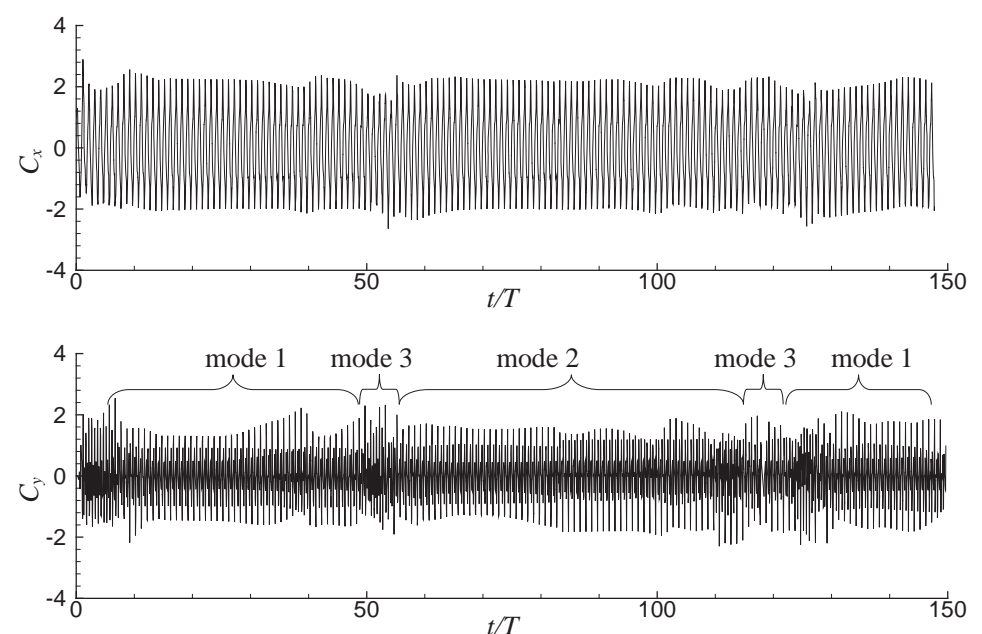

Figure 28. The time histories of $C_{x}$ and $C_{y}$ of $K C=20$ (2D simulation results).
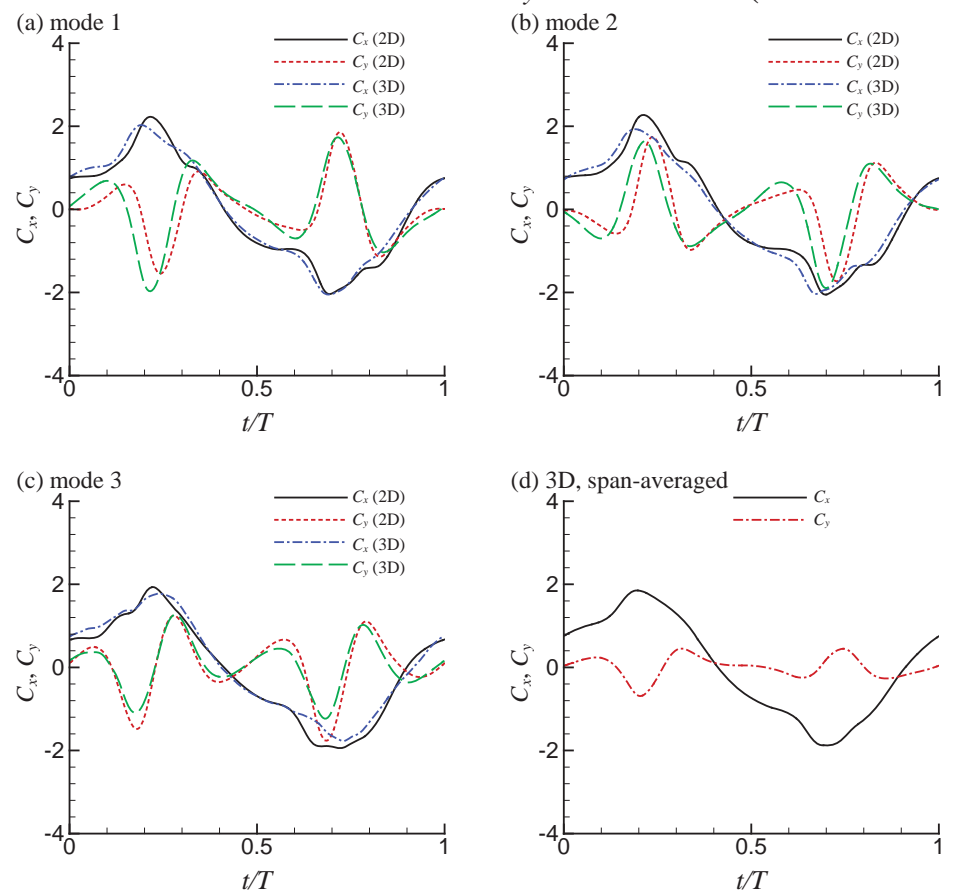

Figure 29. A comparison between the two-dimensional, sectional and span-averaged $C_{x}$ and $C_{y}$ at $K C=20$.
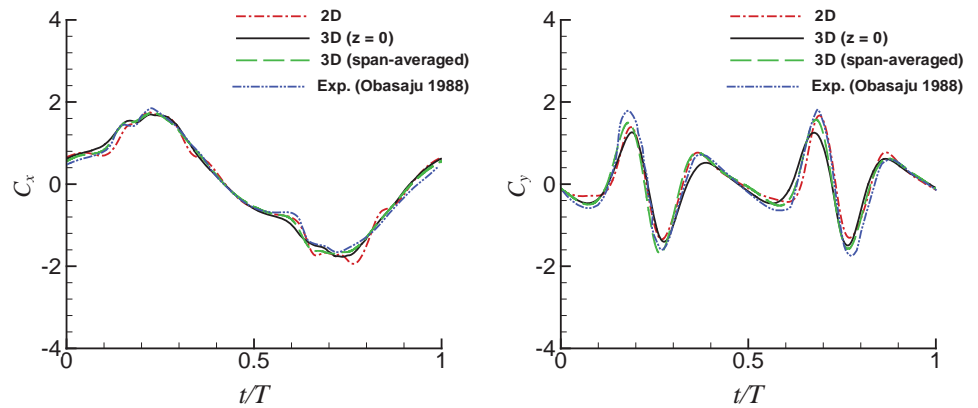

Figure 30. A comparison between the two-dimensional, sectional and span-averaged $C_{x}$ and $C_{y}$ at $K C=26.2$. 

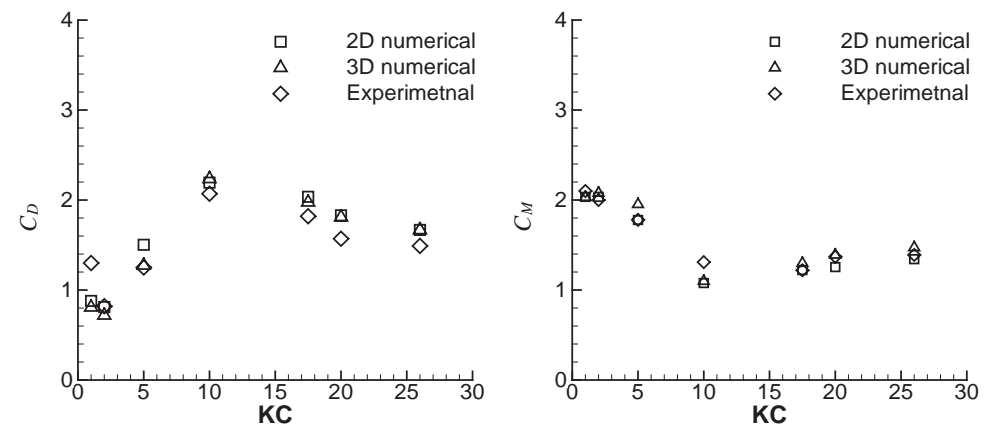

Figure 31. A comparison of Morison coefficients between the 2D, 3D numerical model and experimental data (the corresponding experimental flow conditions (KC, Re) = (1, 2070), (2, 1380), (5, 2415), (10, 1960), (17.5, 1907.5), (20, 2180), (26.2, 2855.8), KC = 1 and 2 were from Sarpkaya (1986) and the other five KC numbers are from Obasaju et al. (1988)) 